\title{
Mechanics of Rotating Isolated Horizons
}

\author{
Abhay Ashtekar ${ }^{1,4 *}$, Christopher Beetle ${ }^{1,2 \dagger}$, and Jerzy Lewandowski ${ }^{3,4 \ddagger}$ \\ 1. Center for Gravitational Physics and Geometry \\ Physics Department, Penn State, University Park, PA 16802, U.S.A. \\ 2. Physics Department, University of Utah, Salt Lake City, Utah 84112 \\ 3. Institute of Theoretical Physics, Warsaw University, ul. Hoża 69, 00-681, Warsaw, Poland \\ 4. Max Planck Institut für Gravitationsphysik, Am Mühlenberg, D14476 Golm, Germany
}

\begin{abstract}
Black hole mechanics was recently extended by replacing the more commonly used event horizons in stationary space-times with isolated horizons in more general space-times (which may admit radiation arbitrarily close to black holes). However, so far the detailed analysis has been restricted to nonrotating black holes (although it incorporated arbitrary distortion, as well as electromagnetic, Yang-Mills and dilatonic charges). We now fill this gap by first introducing the notion of isolated horizon angular momentum and then extending the first law to the rotating case.
\end{abstract}

*E-mail: ashtekar@gravity.phys.psu.edu

${ }^{\dagger}$ E-mail: beetle@physics.utah.edu

${ }^{\ddagger}$ E-mail:jerzy.lewandowski@fuw.edu.pl 


\section{INTRODUCTION}

The zeroth and first laws of black hole mechanics apply to equilibrium situations and small departures therefrom. In standard formulations of these laws, black holes in equilibrium are represented by stationary space-times with regular event horizons (see, e.g., [1]). While this idealization is a natural starting point, from a physical perspective it seems quite restrictive. (See [2,3] for a detailed discussion.) To overcome this limitation, a new model for a black hole in equilibrium was introduced in [2,3]. The generalization is two-fold. First, one replaces the notion of an event horizon with that of an isolated horizon. While the former are defined only retroactively using the fully evolved space-time geometry, the latter are defined quasi-locally by suitably constraining the geometry of the horizon surface itself. Second, one drops the requirement that the space-time be stationary and asks only that the horizon be isolated. That is, the requirement that the black hole be in equilibrium is incorporated by demanding only that no matter or radiation fall through the horizon although the exterior space-time region may well admit radiation. Consequently, the generalization in the class of allowed space-times is enormous. In particular, space-times admitting isolated horizons need not possess any Killing vector field; although event horizons of stationary black holes are isolated horizons, they are a very special case. A recent series of papers [3, 4] has generalized the laws of black hole mechanics to this broader context. The notion of isolated horizons has proved to be useful also in other contexts ranging from numerical relativity to background independent quantum gravity: i) it plays a key role in an ongoing program for extracting physics from numerical simulations of black hole mergers [5 7]; ii) it has led to the introduction [4,8,9] of a physical model of hairy black holes, systematizing a large body of results on properties of these black holes which has accumulated from a mixture of analytical and numerical investigations; and, iii) it serves as a point of departure for statistical mechanical entropy calculations in which all non-rotating black holes (extremal or not) and cosmological horizons are incorporated in a single stroke [2, 10,11].

The first treatment of black hole mechanics using isolated horizons [3] only considered undistorted, non-rotating horizons. That is, the boundary conditions it used imply the intrinsic geometry of the horizon is spherically symmetric and the imaginary part of the Weyl curvature component $\Psi_{2}$ - which encodes gravitational angular momentum-vanishes at the horizon. Although they do not constrain fields in the exterior region in any way (even close to the horizon), these restrictions are, nonetheless, very strong. More recently, these boundary conditions were significantly weakened to allow for both distortion and rotation and the basic, geometric consequences of the more general boundary conditions were analyzed [4. The zeroth law of black hole mechanics was also extended to the more general context, and the first law, to non-rotating but possibly distorted isolated horizons. The purpose of this paper is to extend the first law to the rotating case. We will first introduce (quasi-local) definitions of angular momentum and mass of the isolated horizon in this context and then establish the first law. Thus, this paper is a continuation of [4 and completes the task of deriving the black hole mechanics of all isolated horizons of direct physical interest.

Let us outline the key new points which distinguish the rotating case considered here. First, if the (gravitational contribution to the) horizon angular momentum is to be non-zero, the Weyl component $\operatorname{Im}\left[\Psi_{2}\right]$ cannot vanish on the horizon. Therefore, we will extend the 
Hamiltonian framework of [4] by lifting the restriction on $\operatorname{Im}\left[\Psi_{2}\right]$. Second, by analogy with Killing horizons, in the non-rotating case it was natural to require the time-evolution vector field to lie along the null normal to the horizon. In the general rotating case, by contrast, we expect the natural "time-translation-like" vector field to be space-like on the horizon, with components both along the null generators and along a transverse "rotational" direction. Our evolution field will have this feature. Third, to have a well-defined notion of angular momentum, one expects there should exist a rotational symmetry on the horizon. We will analyze symmetries of all isolated horizons and show that the boundary conditions imply that there are three universal classes, characterized by the structure of the symmetry group: I) spherically symmetric horizons; II) axi-symmetric horizons; and, III) general, distorted horizons (with no symmetry other than that along the null generators). To have a welldefined notion of angular momentum, we will focus on class II. (Class I was discussed in [3] while the focus of [4 was on non-rotating isolated horizons in class III.)

In spite of these differences, the basic techniques used in this paper are very similar to those of [4]. The Hamiltonian formalism is again employed to motivate the definition of horizon mass and, in our rotating case, also angular momentum. The first law again emerges as a necessary and sufficient condition for the evolution to be Hamiltonian, but now acquires new terms resulting from the angular momentum of the horizon.

The plan of this paper is as follows. Section [1] briefly recalls the definition and basic structure of isolated horizons as discussed in [4]. Section [II] analyzes the possible symmetries of isolated horizons. Section $\mathbb{D}$ proves the first law of black hole mechanics for space-times containing rotating isolated horizons. Section $\mathrm{V}$ summarizes the results and the Appendix discusses the issue of defining Hamiltonians generating diffeomorphisms which need not be horizon symmetries.

\section{PRELIMINARIES}

This section summarizes the basic properties of isolated horizons and introduces the notation used in this paper. Specifically, subsection [IA recalls from [4 the definition of a weakly isolated horizon in Einstein-Maxwell theory and several of its immediate consequences. Subsection II B analyzes certain geometric structures on the horizon. This discussion is essential to the classification of horizon symmetries presented in section III and has not appeared before. Finally, subsection II ra reviews the covariant phase space of space-times admitting weakly isolated horizons as inner boundaries, constructed in [4].

Let us begin fixing a few conventions. Throughout this paper, we assume all manifolds and fields are smooth and restrict ourselves to the Einstein-Maxwell theory. Space-time $\mathcal{M}$ is a 4 -dimensional manifold equipped with a metric $g_{a b}$ of signature $(-,+,+,+)$ and a Maxwell potential $\mathbf{A}_{a}$. If $\Delta$ is a null hypersurface in $\left(\mathcal{M}, g_{a b}\right)$, its future-directed null normal direction will be denoted $\left\{\ell^{a}\right\}$. This direction field is naturally an equivalence class of vector fields on $\Delta$ under rescaling by arbitrary positive functions. We refer to it simply as the null normal to $\Delta$. Pull-backs to $\Delta$ of the covariant tensor fields on $\mathcal{M}$ (or more generally, covariant indices of arbitrary tensor fields) will be denoted by an under-arrow and equalities restricted to $\Delta$, by the symbol $\widehat{=}$. The (degenerate) intrinsic geometry on $\Delta$ is described by the metric $q_{a b}: \widehat{=} g_{a b}$. A tensor $q^{a b}$ on $\Delta$ will be said to be an "inverse" of $q_{a b}$ if it satisfies 
$q^{a b} q_{a c} q_{b d} \widehat{=} q_{c d}$. Because of the degeneracy of the intrinsic geometry on $\Delta$, the inverse metric is not unique, but can be changed freely by the addition of a term of the form $V^{(a} \ell^{b)}$ with $V^{a}$ tangent to $\Delta$ and $\ell^{b} \in\left\{\ell^{b}\right\}$. The expansion $\theta_{(\ell)}$ of the null normal field $\ell^{a} \in\left\{\ell^{a}\right\}$ is defined by $\theta_{(\ell)}:=q^{a b} \nabla_{a} \ell_{b}$, where $\nabla_{a}$ is the torsion-free connection on $\mathcal{M}$ defined by $g_{a b}$. It is straightforward to check this definition is independent of the choice of inverse metric, but does depend upon the choice of null normal vector.

\section{A. Weakly Isolated Horizons}

A weakly isolated horizon consists of a pair $\left(\Delta,\left[\ell^{a}\right]\right)$, where $\Delta$ is a 3-dimensional submanifold of $\mathcal{M}$ and $\left[\ell^{a}\right]$ is an equivalence class of vector fields on $\Delta$ under constant rescalings, such that

(i) $\Delta$ is topologically $S^{2} \times \mathrm{R}$ and null, [ $\left.\ell^{a}\right]$ lies along its null normal and the space of its integral curves is diffeomorphic to $S^{2}$;

(ii) The expansion $\theta_{(\ell)}$ of $\Delta$ vanishes for any choice $\ell^{a} \in\left[\ell^{a}\right]$ of the null normal;

(iii) The space-time connection has a (partial) symmetry along $\left[\ell^{a}\right]$ in the sense that

$$
\left[\mathcal{L}_{\ell}, \nabla_{a}\right] \ell^{b} \widehat{=} 0
$$

for any choice of $\ell^{a} \in\left[\ell^{a}\right]$;

(iv) All equations of motion hold at $\Delta$; and,

(v) The Maxwell potential $\mathbf{A}$ is gauge-adapted to the horizon in the sense that

$$
\mathcal{L}_{\ell} \mathbf{A}_{\leftarrow}=0
$$

for any choice of $\ell^{a} \in\left[\ell^{a}\right]$.

A Killing horizon (with topology $S^{2} \times \mathrm{R}$ ) in Einstein-Maxwell theory is automatically a weakly isolated horizon under this definition, provided the Maxwell field strength is symmetric along the Killing field and the gauge of its potential is chosen to satisfy (2.2). If the Killing field is defined only in a neighborhood of the horizon, there is no obvious way to fix the freedom of rescaling it by a constant. This freedom is reflected in our freedom to rescale $\ell$ by a constant. Note, however, the definition admits a much broader class of examples. To explore this class, let us begin by examining the motivations behind the conditions themselves.

Some of the restrictions made by the above conditions are relatively tame. For example, the topological requirement in condition (ii) simply restricts the horizon to have the topology which one expects to arise from gravitational collapse. This restriction can be weakened to allow for more general topologies [4.12], though we shall not discuss this possibility further here. More importantly, the remainder of condition (1) makes clear the roles of $\Delta$ and $\left[\ell^{a}\right]$ : $\Delta$ is the horizon surface and $\left[\ell^{a}\right]$ is a distinguished class of its null normals. Condition (iv) is 
also straightforward; it applies a dynamical restriction closely analogous to the one usually imposed at infinity. However, while the metric at infinity is required to approach a specific asymptotic solution to the Einstein equations, condition (iv) allows the metric to approach any solution to the Einstein equations at the horizon.

Conditions (i) and (iv) are satisfied on a wide variety of surfaces, including many even in Minkowski space-time. However, the vast majority of these surfaces do not have the characteristics one would intuitively expect on a "horizon." The first key condition which distinguishes a weakly isolated horizon is (iii). It implies the cross-sectional area of $\Delta$ is constant "in time," thereby capturing the notion of isolation without introducing a Killing field. The horizon area is denoted $a_{\Delta}$ and we define its radius $R_{\Delta}$ by

$$
a_{\Delta}=4 \pi R_{\Delta}^{2} .
$$

This condition makes the definition dramatically stronger. For example, it implies there are in fact no weakly isolated horizons in Minkowski space-time.

To discuss condition (iii), it will be useful to first explore some consequences of condition (iii) by itself. First, since $\ell^{a} \in\left[\ell^{a}\right]$ is normal to $\Delta$, it is automatically twist-free and geodetic:

$$
\ell^{a} \nabla_{a} \ell^{b} \widehat{=} \kappa_{(\ell)} \ell^{b}
$$

Motivated by the usual definition for Killing horizons, the acceleration $\kappa_{(\ell)}$ of $\ell^{a}$ will be called the surface gravity of $\Delta$ with respect to the normal $\ell^{a}$. As one can see from (2.3), when $\ell^{a}$ is rescaled within $\left[\ell^{a}\right]$, the surface gravity scales by the same factor. Thus, the surface gravity of a weakly isolated horizon is generally defined only up to a positive constant multiplicative factor. Second, given the vanishing twist and expansion of $\ell^{a}$, one can use the Raychaudhuri equation for the null congruence generated by $\ell^{a}$ to conclude its shear must also vanish, and that

$$
R_{a b} \ell^{a} \ell^{b} \hat{=} 0
$$

Third, the vanishing twist, shear and expansion of $\ell^{a}$ imply the existence of a 1-form $\omega_{a}$, intrinsic to $\Delta$, satisfying

$$
\nabla_{a} \ell^{b} \widehat{=} \omega_{a} \ell^{b}
$$

This 1 -form $\omega_{a}$ is independent of the choice of $\ell^{b} \in\left[\ell^{b}\right]$ and its contraction with $\ell^{a}$ will yield the surface gravity $\kappa_{(\ell)}$ defined above. Furthermore, as we will now discuss, condition (iii) causes $\omega_{a}$ to play a central role in the theory of weakly isolated horizons.

One important consequence of (2.5) is that $\ell^{a}$ is a symmetry of the degenerate intrinsic geometry of $\Delta$ in the sense that

$$
\mathcal{L}_{\ell} q_{a b} \widehat{=} 0 \text {. }
$$

In the case of a non-null hypersurface in space-time on which the intrinsic geometry is nondegenerate, the existence of a Killing vector for the intrinsic metric would automatically imply the intrinsic connection was likewise preserved along that Killing vector. In other words, for a non-degenerate intrinsic geometry, the restriction (2.1) of condition (iii) would 
follow from (2.6). However, this is not the case for a null hypersurface since the intrinsic metric does not determine the intrinsic connection uniquely. Thus, condition (iiii) must be imposed separately. The logic of making this restriction, however, is clear: we are extending the symmetry (2.6) of the degenerate metric to the intrinsic connection on $\Delta$ given by $\nabla_{a}$. Note, however, that condition (iii) does not restrict the entire connection on $\Delta$, but only its

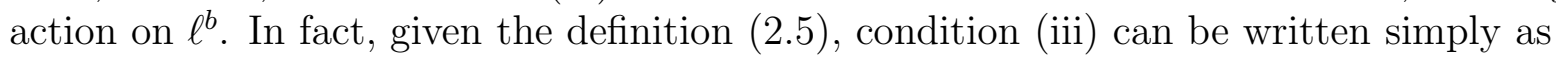

$$
\mathcal{L}_{\ell} \omega_{a}=0 .
$$

This formula is much easier to apply in practice than (2.1), which is why the 1-form $\omega_{a}$ plays an important role in our formulation. (If one requires that $\ell^{a}$ be a symmetry of the full pull-back of the connection to $\Delta$, we obtain isolated horizons [4.6.6. While this stronger condition is physically reasonable, it is significantly more difficult to check its validity in examples. Since the stronger condition is not needed in our proof of the zeroth and the first laws, we have chosen to work with weakly isolated horizons.)

Finally, let us examine condition ( $\mathbb{\nabla}$ ) on the Maxwell field. At first, the symmetry requirement (2.2) on the Maxwell field appears similar to the restriction (2.7) placed on the

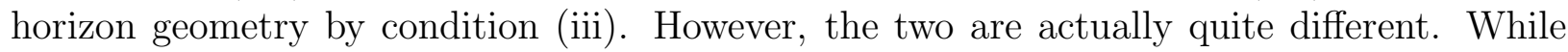
(2.7) represents a genuine restriction on the physical fields at the horizon, (2.2) can always be achieved via a gauge transformation when the other conditions are satisfied. Using the Einstein equations at the horizon and the consequence (2.4) of the Raychaudhuri equation one can show the 1-form $\ell^{a} \mathbf{F}_{a b}$ is null at the horizon. Since this 1-form is also orthogonal to $\ell^{b}$, it must be proportional to $\ell_{b}$, whence円

$$
\ell\lrcorner \underset{\leftarrow}{\mathbf{F}} \hat{=} 0 .
$$

Then, using the Cartan formula for the Lie derivative and the Maxwell equations at the horizon, one finds

$$
\left.\mathcal{L}_{\ell} \underset{\leftarrow}{F}=\ell\right\lrcorner \underset{\mathrm{d} F}{\longleftarrow}+\underbrace{}_{\mathrm{d}(\ell\lrcorner \mathbf{F})}=0 .
$$

Thus, because the field strength is already compatible with the symmetry (2.2) imposed on the Maxwell potential by condition ( $\mathrm{V}$ ), (2.2) is indeed merely a (partial) choice of gauge.

We conclude this subsection with several remarks. Each of these points is discussed in more detail in [4].

First, while this paper explicitly examines only those weakly isolated horizons which occur in Einstein-Maxwell theory, it is not difficult to generalize the definition given here to allow other matter fields near the horizon. However, it is not possible to incorporate arbitrary types of matter, but only those which satisfy a certain energy condition. Specifically, the stress-energy tensor of each matter field present at the horizon must have the property that $T_{a b} \ell^{b}$ is future directed and causal] for any $\ell^{b} \in\left[\ell^{b}\right]$. This condition follows immediately from

\footnotetext{
${ }^{1}$ The symbol $\left.\cdot\right\lrcorner$ represents the contraction of a vector field on the first index of a differential form.

${ }^{2} \mathrm{~A}$ vector is said to be causal if it is either time-like or null.
} 
the dominant energy condition which is usually assumed in proofs of the laws of black hole mechanics. Moreover, since the Maxwell (or Yang-Mills, etc.) field satisfies the dominant energy condition, it will automatically meet the (weaker) demand made here. This condition is essential to the derivation of (2.4) from the Raychaudhuri equation and to the associated proof that the horizon is shear-free.

Second, the conditions given above imply the space-time geometry at a weakly isolated horizon is algebraically special. This is an interesting feature which carries over from the stationary context where the Kerr-Newman black hole space-times are (globally) of Petrov type II-II. However, there are several important differences. One can show $\left[\ell^{a}\right]$ is a repeated principal null direction at the horizon, whence the space-time geometry is necessarily of Petrov type II there. However, it is not generally possible to identify a second repeated principal null direction at such a horizon, so the geometry needn't be of type II-II. Moreover, the space-time geometry away from the horizon may not be algebraically special at all; our conditions constrain only the local, horizon geometry. Since $\left[\ell^{a}\right]$ is a repeated principal null direction for the space-time metric at the horizon, the Newman-Penrose curvature components satisfy

$$
\Psi_{0} \widehat{=} \Psi_{1} \widehat{=} 0
$$

in any null frame which includes an element $\ell^{a} \in\left[\ell^{a}\right]$ at $\Delta$. It then follows that the NewmanPenrose component $\Psi_{2}$ is "gauge-invariant" (i.e., independent of the other three null tetrad elements) at $\Delta$. In particular, the imaginary part of $\Psi_{2}$ can be expressed without reference to any null tetrad using the 1 -form $\omega_{a}$ introduced in (2.5):

$$
\mathrm{d} \omega=2 \operatorname{Im}\left[\Psi_{2}\right]^{2} \epsilon,
$$

where ${ }^{2} \epsilon_{a b}$ denotes the natural area 2-form on $\Delta$ (see the next subsection).

Third, the conditions enable a simple proof of the zeroth law of black hole mechanics for generic weakly isolated horizons. Examining the definition (2.3) of surface gravity for a weakly isolated horizon and the definition (2.5) of $\omega_{a}$, one finds

$$
\kappa_{(\ell)}=\ell^{a} \omega_{a}
$$

whence (by the Cartan formula) we have

$$
\left.\left.\mathrm{d} \kappa_{(\ell)} \equiv \mathrm{d}(\ell\lrcorner \omega\right)=\mathcal{L}_{\ell} \omega-\ell\right\lrcorner \mathrm{d} \omega .
$$

The first term on the right hand side vanishes because of (2.7), while the second vanishes because $\mathrm{d} \omega$ is given by $(2.9)$ and the contraction of ${ }^{2} \epsilon_{a b}$ with $\left[\ell^{a}\right]$ vanishes. Thus, the surface gravity of a weakly isolated horizon is indeed constant; the zeroth law holds. Note, however, that because $\Delta$ is equipped only with an equivalence class $\left[\ell^{a}\right]$ of null normals, we have the constant rescaling freedom $\ell^{a} \rightarrow \tilde{\ell}^{a}=c \ell^{a}$ under which the surface gravity transforms via $\kappa_{(\ell)} \rightarrow \kappa_{(\tilde{\ell})}=c \kappa_{(\ell)}$. Therefore, while surface gravity is constant on an isolated horizon, we can not assign a particular value to it. This is not surprising: one cannot assign a specific numerical value to surface gravity even on a locally defined Killing horizon, since the Killing field is defined only up to rescaling by a constant. Finally, we note the argument establishing the zeroth law for weakly isolated horizons can also be applied to the Maxwell field. Repeating this argument, with $\omega$ replaced by A, and applying (2.2) and (2.8) one 
finds the quantity $\ell\lrcorner \mathbf{A}$ is also constant over the horizon surface. Motivated by this fact, we define the electric potential of the horizon by

$$
\Phi_{(\ell)}:=-\ell^{a} \mathbf{A}_{a}
$$

Once again, while the electric potential is always constant over $\Delta$, its value is not fixed.

\section{B. Geometrical Structures}

We will first discuss the relation between certain types of fields on $\Delta$ and fields on the 2-sphere $\widehat{\Delta}$ of integral curves of $\left[\ell^{a}\right]$ and then show that if $\kappa_{(\ell)}$ is non-zero, $\Delta$ admits a natural foliation. This discussion will be useful especially in section [II] for our analysis of the symmetry algebras of weakly isolated horizons.

Denote by $\mathcal{P}$ the natural projection map $\mathcal{P}$ from $\Delta$ to $\widehat{\Delta}$. A vector field $W^{a}$ defined intrinsically on $\Delta$ can be unambiguously projected to a vector field $\widehat{W}^{a}$ on $\widehat{\Delta}$ if and only if $\mathcal{L}_{\ell} W^{a}$ is proportional to $\ell^{a}$. Similarly, a covariant tensor field $T_{a_{1} \cdots a_{n}}$ on $\Delta$ is a pull-back under $\mathcal{P}$ of a tensor field $\widehat{T}_{a_{1} \cdots a_{m}}$ on $\widehat{\Delta}$ if and only if two conditions hold: i) $T_{a_{1} \cdots a_{m}}$ is transversal to $\ell$ in the sense that the contraction of any of its indices with $\ell$ vanishes; and, ii) the Lie derivative $\mathcal{L}_{\ell} T_{a_{1} \cdots a_{m}}$ vanishes. These are rather general properties of manifolds ruled by one-dimensional curves.

Applying them to the case of a weakly isolated horizon, we conclude that the degenerate metric $q_{a b}$ is the pull-back to $\Delta$ of a Riemannian metric $\hat{q}_{a b}$ on $\widehat{\Delta}$. The connection 1 -form $\omega_{a}$ does satisfy condition ii) above. However, since

$$
\ell^{a} \omega_{a} \widehat{=} \kappa_{(\ell)}
$$

it defines a 1 -form $\widehat{\omega}_{a}$ on $\widehat{\Delta}$ only in the extremal case, i.e., only when $\kappa_{(\ell)} \widehat{=} 0$. Finally, the projection map $\mathcal{P}$ enables us to define a preferred area element ${ }^{2} \epsilon_{a b}$ on $\Delta$. Using the Riemannian metric $\widehat{q}_{a b}$, one can construct the area element $\hat{\epsilon}_{a b}$ on $\widehat{\Delta}$ and pull that 2-form back to $\Delta$ under $\mathcal{P}$. By construction, the resulting 2 -form ${ }^{2} \epsilon_{a b}$ on $\Delta$ satisfies

$$
\ell^{a} \epsilon_{a b}=0 \quad \text { and } \quad \mathcal{L}_{\ell}^{2} \epsilon_{a b}=0
$$

The first of these results was already used in the proof of the zeroth law in the last subsection.

Let us now assume that $\kappa_{(\ell)}$ is non-zero and discuss the preferred foliation of $\Delta$ (for further details and a discussion of global subtleties, see [6]). For simplicity, consider first the "non-rotating case" where $\operatorname{Im}\left[\Psi_{2}\right]=0$. According to (2.9), this is precisely the case where the connection form $\omega_{a}$ is curl-free. Hence $\omega=\mathrm{d} v$ for some function $v$ on $\Delta$. Furthermore, $\ell\lrcorner \mathrm{d} v=\kappa_{(\ell)}$ is a non-zero constant on $\Delta$, whence $\left[\ell^{a}\right]$ is transverse to the $v=$ const surfaces. Thus, $v=$ const surfaces define a natural foliation of $\Delta$ by a family of 2 -spheres.

To conclude, let us consider the rotating case. While $\operatorname{Im}\left[\Psi_{2}\right]$ no longer vanishes in this case, it is still constant along each generator of $\Delta$, i.e., is a pull-back of a function $\operatorname{Im}\left[\Psi_{2}\right]$ on $\widehat{\Delta}$. Using (2.9) as the motivation, let us introduce a 1-form $\widehat{\alpha}_{a}$ on $\widehat{\Delta}$ such that $\widehat{\mathrm{d}} \widehat{\alpha}=2 \operatorname{Im} \widehat{\left[\Psi_{2}\right]} \widehat{\epsilon}$. Of course, this potential $\widehat{\alpha}_{a}$ is not unique; one is free to add to it the gradient of any function on $\widehat{\Delta}$. There is, however, a natural gauge condition which fixes this 


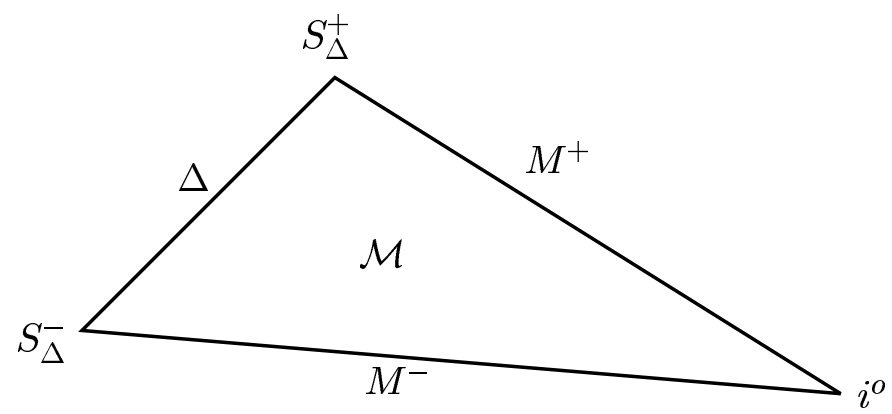

FIG. 1. The region of space-time $\mathcal{M}$ under consideration has an internal boundary $\Delta$ and is bounded by two partial Cauchy surfaces $M^{ \pm}$which intersect $\Delta$ in the 2-spheres $S_{\Delta}^{ \pm}$and extend to spatial infinity $i^{o}$.

ambiguity: Since there are no harmonic 1 -forms on the 2 -sphere $\widehat{\Delta}$, there exists a unique 1-form $\hat{\alpha}_{a}$ such that

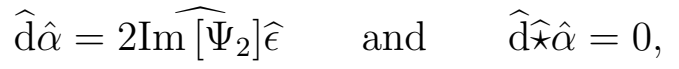

where $\widehat{\epsilon}_{a b}$ is the area element on $\widehat{\Delta}$ and $\widehat{\star}$ denotes the Hodge dual on $\widehat{\Delta}$. Let $\alpha_{a}$ be the pull-back to $\Delta$ of $\hat{\alpha}_{a}$ and set

$$
\tilde{\omega}_{a}=\omega_{a}-\alpha_{a} .
$$

Then, $\mathrm{d} \tilde{\omega}_{a}=0$ and $\ell^{a} \tilde{\omega}_{a}=\kappa_{(\ell)}$ on $\Delta$. Thus, $\tilde{\omega}$ has the same properties that $\omega_{a}$ had in the nonrotating case. Therefore, it defines a foliation of $\Delta$ by a family of 2 -spheres. Our gauge choice is natural in the sense that it is the only choice for which this more general construction, when applied to the non-rotating case, reproduces the natural foliation obtained above. (Indeed, in the non-rotating case and in our gauge, $\hat{\alpha}_{a}$ vanishes on $\widehat{\Delta}$ and $\tilde{\omega}_{a}=\omega_{a}$ on $\Delta$.) While this foliation is used only to elucidate certain points in this paper, it plays a key role in the analysis of the near-horizon, strong field geometry [7] and in extracting physics from space-times obtained via numerical simulations, such as those associated with black hole mergers [7, 5].

\section{Covariant Phase Space}

In the spirit of [4], we will use Hamiltonian methods to introduce notions of angular momentum and mass of weakly isolated horizons. In this sub-section, we recall from [4] the structure of the underlying phase space.

The most interesting space-times containing weakly isolated horizons are of the black hole type, so we will tailor the topological structure of the underlying space-time manifold to this case. Specifically, fix a manifold $\tilde{\mathcal{M}}$ with boundary where the boundary consists of four components: an internal boundary $\tilde{\Delta}$, topologically $S^{2} \times \mathrm{R}$ which will finally serve as the isolated horizon, an outer boundary $\tau_{\infty}$, also topologically $S^{2} \times \mathrm{R}$ which will serve as a time-like cylinder at spatial infinity, and two surfaces $M^{ \pm}$, which are intended to be space-like and to serve as future and past boundaries of $\mathcal{M}$. The intersections of $M^{ \pm}$with 
$\Delta$ and $\tau_{\infty}$ will be denoted $S_{\Delta}^{ \pm}$and $S_{\infty}^{ \pm}$, respectively (see FIG 1 ). The inner boundary $\Delta$ will be equipped with a privileged equivalence class $\left[\ell^{a}\right]$ of vector fields under constant rescalings, whose integral curves constitute a 2 -sphere $\vec{\Delta}$. In the Hamiltonian framework, we will not be interested in the surfaces $M^{ \pm}$and $\tau_{\infty}$. Therefore, it is convenient to introduce $\mathcal{M}:=\tilde{\mathcal{M}}-\left(M^{+} \cup M^{-} \cup \tau_{\infty}\right)$ and $\Delta:=\tilde{\Delta}-\left(S_{\Delta}^{+} \cup S_{\Delta}^{-}\right)$.

We will consider Einstein-Maxwell theory in a first-order framework based on tetrads. Therefore, our basic physical fields will consist of a triple $\left(e_{I}^{a}, A_{a I}{ }^{J}, \mathbf{A}_{a}\right)$ on $M$, where $e_{I}^{a}$ is a tetrad on space-time, $A_{a I}{ }^{J}$ is the connection form (relative to a fiducial flat connection $\partial_{a}$ ) for a connection $D_{a}$ in the frame bundle over space-time, and $\mathbf{A}_{a}$ is a Maxwell connection on a trivial $U(1)$ bundle over $\mathcal{M}$ (see [4] $)$. (Whenever needed, these fields will be extended to the boundaries $S_{\Delta}^{ \pm}$of $\Delta$ by continuity.) These fields are subject to certain boundary conditions: We require that each space-time $\left(\mathcal{M}, e_{I}^{a}\right)$ is asymptotically flat at spatial infinity and admits $\left(\Delta,\left[\ell^{a}\right]\right)$ as a weakly isolated horizon. For convenience, we will fix an internal tetrad $\left(\ell^{I}, n^{I}, m^{I}, \bar{m}^{I}\right)$ at each point of $\Delta$ and require the tetrad field to satisfy $\ell^{I} e_{I}^{a} \in\left[\ell^{a}\right]$ on $\Delta$. (This can always be achieved and serves to eliminate an irrelevant part of the tetradrotation freedom.)

With this kinematical structure at hand, we can now specify the action functional:

$$
S[e, D, \mathbf{A}]=\frac{-1}{16 \pi G} \int_{\mathcal{M}} \operatorname{Tr}[\Sigma \wedge F]+\frac{1}{16 \pi G} \int_{\tau_{\infty}} \operatorname{Tr}[A \wedge \Sigma]-\frac{1}{8 \pi} \int_{\mathcal{M}} \mathbf{F} \wedge \star \mathbf{F},
$$

where the traces are taken over the internal (tetrad) indices, $F_{a b I}{ }^{J}$ denotes the curvature of the connection $A_{a I}{ }^{J}$, and the 2-forms $\Sigma_{a b I}{ }^{J}$ are defined in terms of the (co-)tetrad field by

$$
\Sigma_{a b I}{ }^{J}:=\epsilon_{I}^{J} K L e_{a}^{K} e_{b}^{L} .
$$

Interestingly, while the asymptotic boundary term is needed to make the action principle well-posed given the asymptotically flat fall-off conditions imposed near infinity, no such boundary term is needed at the horizon surface $\Delta$. (For details, see $\|4\|$.)

Our covariant phase space $\mathbb{\Gamma}$ will consist of the space of solutions $\left(e_{I}^{a}, A_{a I}{ }^{J}, \mathbf{A}_{a}\right)$ to the Einstein-Maxwell equations, satisfying the boundary conditions specified above. As usual, the symplectic structure is constructed on $\mathbb{\Gamma}$ using the (anti-symmetrized) second variation of the action (2.13). Applying the equations of motion to this second variation, one discovers the integral over $\mathcal{M}$ reduces to surface terms at $M^{ \pm}$and at $\Delta$ (the surface term at $\tau_{\infty}$ vanishes because of the asymptotic fall-off conditions). In order to reduce the expression for the second variation further, it is convenient to introduce a pair of scalar functions on $\Delta$ which act as "potentials" for the surface gravity and electric potential. Given any point $\left(e_{I}^{a}, A_{a I}{ }^{J}, \mathbf{A}_{a}\right)$ in phase space, the scalar field $\psi$ is defined by

$$
\psi=0 \quad \text { on } S_{\Delta}^{-} \text {, and } \quad \mathcal{L}_{\ell} \psi=\kappa_{(\ell)}
$$

and the scalar field $\chi$ by

$$
\chi=0 \quad \text { on } S_{\Delta}^{-} \text {, and } \quad \mathcal{L}_{\ell} \chi=-\Phi_{(\ell)} .
$$

Note that both of these functions are completely determined by the physical fields. (A priori there is some freedom in the choice of initial values of $\psi$ and $\chi$ on $S_{\Delta}^{-}$. While the symplectic 
structure is sensitive to these choices, none of the final results are.) Expressed in terms of $\psi$ and $\chi$, the surface term at $\Delta$ in the second variation of the action turns out to be exact and thus reduces to a pair of integrals on $S_{\Delta}^{ \pm}$. The integral over $M^{-}$, together with its surface term at $S_{\Delta}^{-}$is then taken to define the symplectic structure for the theory. In fact, when the equations of motion hold and both variations satisfy the linearized equations of motion, the integral which defines the symplectic structure may be taken over any partial Cauchy surface $M$ in $\mathcal{M}$. It is given by

$$
\begin{aligned}
\Omega\left(\delta_{1}, \delta_{2}\right)= & \frac{-1}{16 \pi G} \int_{M} \operatorname{Tr}\left[\delta_{1} A \wedge \delta_{2} \Sigma-\delta_{2} A \wedge \delta_{1} \Sigma\right]-\frac{1}{8 \pi G} \oint_{S_{\Delta}} \delta_{1} \psi \delta_{2}{ }^{2} \epsilon-\delta_{2} \psi \delta_{1}{ }^{2} \epsilon \\
& +\frac{1}{4 \pi} \int_{M} \delta_{1} \mathbf{A} \wedge \delta_{2}(\star \mathbf{F})-\delta_{2} \mathbf{A} \wedge \delta_{1}(\star \mathbf{F})-\frac{1}{4 \pi} \oint_{S_{\Delta}} \delta_{1} \chi \delta_{2}(\star \mathbf{F})-\delta_{2} \chi \delta_{1}(\star \mathbf{F}),
\end{aligned}
$$

where $M$ intersects $\Delta$ in $S_{\Delta}$. Details of this result are discussed in [4].

In the covariant Hamiltonian framework, infinitesimal gauge transformations are in 1-1 correspondence with the degenerate directions of the symplectic structure. For example in the asymptotically flat context without internal boundaries, infinitesimal diffeomorphisms and internal Lorentz rotations are in the kernel of the symplectic structure if and only if they vanish asymptotically. Thus, infinitesimal diffeomorphisms which asymptotically approach Killing fields of the flat background metric are not gauge; they are generated by non-trivial Hamiltonians which define the ADM 4-momentum and angular momentum. The situation is similar at the internal boundary $\Delta$. Infinitesimal diffeomorphisms and internal Lorentz rotations need not be in the kernel of the symplectic structure unless they vanish on $\Delta$. Indeed, we will show in Section $\mathbb{\nabla}$ that space-time motions corresponding to time translations and rotations are generated by non-trivial Hamiltonians with surface terms both at infinity and at the horizon. The horizon surface terms will then be interpreted as the horizon energy and angular momentum. However, since infinitesimal internal rotations (and gauge transformations of the Maxwell theory) on $\Delta$ are not automatically in the kernel of the symplectic structure, one must separately ensure that physical quantities such as energy and angular momentum are gauge invariant.

\section{SYMMETRIES OF A WEAKLY ISOLATED HORIZON}

In this section we analyze the possible symmetries of a weakly isolated horizon. Because the generators $\left[\ell^{a}\right]$ of $\Delta$ are not assumed to be complete, we will focus on infinitesimal symmetries. Since the horizon is the inner boundary of space-times under consideration while spatial infinity is the outer, one would expect the horizon symmetries to be analogous to the asymptotic symmetries at spatial infinity. Thus, it is natural to define the horizon symmetry algebra $\mathcal{L}$ to be the quotient of the Lie algebra of all infinitesimal space-time diffeomorphisms which preserve the horizon structure by its sub-algebra consisting of elements which vanish on the horizon. The key question now is: What is the horizon structure relevant for these considerations? It is here that one would expect a significant departure from the situation at spatial infinity. At infinity, all metrics approach a fixed flat metric whence the relevant geometric structure - and hence also the symmetry algebra - is universal; it does not vary

from one space-time to another. At the horizon, by contrast, we are in the strong field 
regime and the near-horizon geometry can vary from one space-time to another. Therefore, we do not expect the relevant horizon structure or the symmetry algebra $\mathcal{L}$ to be universal. Nonetheless, because the horizon is a boundary and all geometries under consideration are subject to the same boundary conditions, one would expect the horizon symmetry algebra to fall in a small number of "universality classes". We will see that this expectation is correct.

To identify the relevant horizon structure, let us return to the definition of weakly isolated horizons and examine the geometric structures that are essential to the definition. First, we have the manifold $\Delta$ and the equivalence class $\left[\ell^{a}\right]$ of null normals. This structure is shared by all weakly isolated horizons. However, to specify the conditions that $\left[\ell^{a}\right]$ must satisfy for $\left(\Delta,\left[\ell^{a}\right]\right)$ to qualify as an isolated horizon, we also introduced "non-universal" fields $\left(q_{a b}, \omega_{a}\right)$ which vary from one point in our covariant phase space to another. 3 Therefore, to construct the algebra $\mathcal{L}$ corresponding a given isolated horizon, it is natural to focus on space-time vector fields whose action preserves $\left(\Delta,\left[\ell^{a}\right], q_{a b}, \omega_{a}\right)$ and factor out by those which vanish on $\Delta$.

Thus, we can ignore the space-time manifold $\mathcal{M}$ and work just with vector fields $W^{a}$ which are tangential to $\Delta$. To qualify as symmetries, they should satisfy the following two conditions:

$$
\mathcal{L}_{W} \ell^{a} \widehat{=} c_{W} \ell^{a} \quad \text { with } c_{W} \text { constant on } \Delta
$$

and,

$$
\mathcal{L}_{W} q_{a b} \widehat{=} 0 \text { and } \mathcal{L}_{W} \omega_{a} \widehat{=} 0 \text {. }
$$

The set of vector fields $W^{a}$ satisfying both (3.1) and (3.2) form a Lie algebra under the usual commutator bracket. This is the symmetry Lie algebra for the horizon in question. We will denote it by $\mathcal{S}$ and refer to these vector fields $W^{a}$ as infinitesimal symmetries of the horizon.

Before analyzing the full algebra, let us note that, irrespective of the specific weakly isolated horizon under consideration, $\mathcal{S}$ is at least one-dimensional: By setting $W^{a}=\ell^{a}$ for any $\ell^{a} \in\left[\ell^{a}\right]$, we see (3.1) and (3.2) are automatically satisfied by virtue of the very definition of weakly isolated horizons. These infinitesimal symmetries preserve each integral curve of $\ell$. More generally, denote by $\mathcal{I}$ all elements $W^{a}$ of $\mathcal{S}$ of the form $W^{a}=f \ell^{a}$ for some function $f$ on $\Delta$. This will be the sub-algebra of all generator-preserving symmetries. Now, given any infinitesimal symmetry $W^{a}$ in $\mathcal{S}$ and an infinitesimal symmetry $f \ell^{a}$ in $\mathcal{I}$, (3.1) implies the commutator $[W, f \ell]^{a}$ is again proportional to $\ell^{a}$ :

$$
[W, f \ell]^{a} \widehat{=}\left(c_{W} f+\mathcal{L}_{W} f\right) \ell^{a},
$$

where $c_{W}$ is the constant appearing in (3.1). Hence, $\mathcal{I}$ is in fact an ideal of the Lie algebra $\mathcal{S}$. Denote by $\hat{\mathcal{S}}$ the quotient Lie algebra: $\hat{\mathcal{S}}=\mathcal{S} / \mathcal{I}$. For reasons explained below, we will refer

\footnotetext{
${ }^{3}$ Recall from Section $\llbracket \mathrm{IA}$ that the essential restrictions are captured by two conditions: i) $\mathcal{L}_{\ell} q_{a b} \widehat{=}$ 0 ; and, ii) $\mathcal{L}_{\ell} \omega_{a} \widehat{=} 0$.
} 
to it as the algebra of projected symmetries. Thus, the algebra $\mathcal{S}$ of infinitesimal horizon symmetries is a semi-direct sumf of $\mathcal{I}$ and $\hat{\mathcal{S}}$.

To determine the structure of $\mathcal{S}$, therefore, it suffices to examine the Lie algebras $\mathcal{I}$ and $\hat{\mathcal{S}}$ separately. Let us begin with the former. Setting $W^{a}=f \ell^{a}$ in (3.1) we obtain:

$$
\mathcal{L}_{\ell} f=C_{f}
$$

where $C_{f}$ is a constant on $\Delta$. The first of the two conditions in (3.2) does not restrict $f$ in any way while the second implies:

$$
\left.0 \widehat{=} \mathcal{L}_{f \ell} \omega \widehat{=} \mathrm{d}(f \ell\lrcorner \omega\right) \widehat{=} \kappa_{(\ell)} \mathrm{d} f .
$$

Therefore, if $\kappa_{(\ell)} \neq 0$, then $f$ must be constant on $\Delta$. In this case the only generatorpreserving symmetries are given by $W^{a} \in\left[\ell^{a}\right]$ and $\mathcal{I}$ is 1-dimensional. On the other hand, if $\kappa_{(\ell)}=0$ (i.e., $(\Delta,[\ell])$ is extremal), (3.4) imposes no condition on $f$. In this case, $f$ has only to satisfy (3.3) and $\mathcal{I}$ is infinite-dimensional

Next, let us examine the quotient Lie algebra $\hat{\mathcal{S}}$. Since every infinitesimal symmetry $W^{a}$ must satisfy (3.1), it can be projected unambiguously to a vector field $\widehat{W}^{a}$ on $\widehat{\Delta}$. We can now use our discussion of Section $\mathbb{I I B}$ on the relation between fields on $\Delta$ with those on the 2-sphere $\widehat{\Delta}$ of its generators to analyze $\hat{\mathcal{S}}$ in terms of these projections $\widehat{W}^{a}$. The first of Eqs (3.2) is satisfied if and only if $\widehat{W}^{a}$ is a Killing field on $\left(\widehat{\Delta}, \widehat{q}_{a b}\right)$ :

$$
\mathcal{L}_{\widehat{W}} \widehat{q}_{a b}=0
$$

Denote by $\mathcal{K}$ the isometry Lie algebra of $\left(\widehat{\Delta}, \widehat{q}_{a b}\right)$. We have shown that $\hat{\mathcal{S}}$ is a sub-algebra of $\mathcal{K}$. In general, it will only be a proper sub-algebra because $W^{a}$ must also satisfy (3.2). Nonetheless, the fact that $\hat{\mathcal{S}}$ is a sub-algebra of $\mathcal{K}$ plays a key role in the classification of $\mathcal{S}$ because the dimension and topology of $\widehat{\Delta}$ imposes severe restrictions on $\mathcal{K}$. On the one hand, every Killing field $\widehat{W}^{a}$ on $\widehat{\Delta}$ is of the form $\hat{\epsilon}^{a b} \partial_{b} \hat{h}$ for some function $\hat{h}$. On the other hand, since all metrics on a 2-sphere are conformal to one another, any Killing field for a generic metric must be a conformal Killing field of a fixed round 2-sphere metric on that sphere. Every conformal Killing vector field $\widehat{W}^{a}$ of a round metric belongs to one of the following classes: (i) a rotation; (ii) a combination of a boost and rotation commuting with each other, with a non zero boost component; and, (iii) a null rotation, the set of zeros of the vector field has exactly one element. Now, in cases (ii) and (iii), all the orbits of the vector field converge to a same point of the sphere at which $\widehat{W}^{a}=0$ (there are two such points in the case (ii)). Since the function $h$ is constant on every orbit, it follows that in these cases it would be constant whence $\widehat{W}^{a}=0$ on the entire sphere. Therefore, a non-trivial Killing vector field $\widehat{W}^{a}$ is of the class (i).

Thus, we conclude that a general metric on a 2-sphere is of one of three types:

\footnotetext{
${ }^{4}$ Had $\Delta$ been complete, we could have integrated $\mathcal{L}$ to obtain a horizon symmetry group $\mathcal{G}$ which would have been a semi-direct product of the groups generated by $\mathcal{I}$ and $\hat{\mathcal{S}}$, the former being the normal sub-group of $\mathcal{G}$.
} 
1. The metric is round; $\mathcal{K}$ is three-dimensional and isomorphic to so(3);

2. The metric is axially symmetric; $\mathcal{K}$ is one-dimensional and isomorphic to $\mathbf{s o}(2)$;

3. The metric has no Killing fields; $\mathcal{K}$ is zero-dimensional (i.e., consists only of the zero element).

In each case, the sub-algebras of $\mathcal{K}$ are easy to characterize. Only in the first case does $\mathcal{K}$ admit a non-trivial sub-algebra which then must be isomorphic to so(2). Hence, the quotient algebra $\hat{\mathcal{S}}$ must be isomorphic either to $\mathbf{s o}(3)$ or $\mathbf{s o}(2)$ or must be the trivial Lie algebra consisting of only of the zero element.

Thus we can divide the set of all weakly isolated horizons into the three classes:

I. Spherically symmetric; the algebra $\hat{\mathcal{S}}$ of projected symmetries is 3 -dimensional. If $\kappa_{(\ell)} \neq 0$, the horizon symmetry algebra $\mathcal{L}$ is the 4 -dimensional semi-direct sum of the additive group $\mathrm{R}$ of reals with $\mathbf{s o}(\mathbf{3})$. If $\kappa_{(\ell)}=0$, then $\mathcal{L}$ is the semi-direct sum of the infinite dimensional Lie algebra defined by (3.3) with so(3).

II. Axi-symmetric; $\hat{\mathcal{S}}$ is 1 -dimensional. If $\kappa_{(\ell)} \neq 0$, the horizon symmetry algebra $\mathcal{L}$ is the 2 -dimensional Abelian Lie algebra generated by vector fields $a \ell^{a}+b \phi^{a}$ where $\phi^{a}$ is a rotation on $\Delta$ and $a, b$ constants. If $\kappa_{(\ell)}=0$, then $\mathcal{L}$ is the semi-direct sum of the infinite dimensional Lie algebra defined by $(3.3)$ with so(2).

III. Generic; $\hat{\mathcal{S}}$ is zero-dimensional. If $\kappa_{(\ell)} \neq 0$, the horizon symmetry group $\mathcal{L}$ is 1 dimensional. If $\kappa_{(\ell)}=0$, then $\mathcal{L}$ is the infinite dimensional Lie algebra defined by (3.3)

As mentioned in the Introduction, in the next section, we will focus on class II.

We will conclude with two remarks.

1. In the above discussion, we have focussed only on symmetries of the relevant horizon geometry. In the Einstein-Maxwell theory, it is natural to require that an infinitesimal symmetry $W^{a}$ of a given weakly isolated horizon also satisfy $\mathcal{L}_{W} F_{a b} \widehat{=} 0$. Physically, this condition requires the symmetry to preserve the flux of the magnetic field through the horizon. It is natural to demand the same of the electric flux. Therefore, we will also insist that $\mathcal{L}_{W}(\star F)_{a b} \widehat{=} 0$ at the horizon. Neither of these additional conditions will affect the classification scheme described above in any way.

2. As shown in Section [IB, if $\kappa_{(\ell)} \neq 0$, the weakly isolated horizon $\left(\Delta,\left[\ell^{a}\right]\right)$ admits a canonical foliation by 2 -spheres $S_{\Delta}$ which can be used to lift vector fields on $\widehat{\Delta}$ to "horizontal" vector fields on $\Delta$. In particular, then, there is a canonical injection of the projective symmetry algebra $\hat{\mathcal{S}}$ into the total symmetry algebra $\mathcal{S}$. As a result, the semi-direct sum structure reduces simply to a direct sum structure. In this case, one can write any symmetry vector $W^{a}$ in the form

$$
W^{a}=B_{W} \ell^{a}+h_{W}^{a}
$$

where $B_{W}$ is a constant and $h_{W}^{a}$ is a horizontal vector field (i.e. tangent to the preferred 2-spheres $S_{\Delta}$ ), satisfying $\mathcal{L}_{\ell} h_{W}^{a}=0$. The condition $\mathcal{L}_{W} \omega_{a}=0$ further implies $\mathcal{L}_{h_{W}} \omega_{a} \widehat{=} 0$, and hence $\mathcal{L}_{h_{W}}\left(\operatorname{Im}[\Psi]_{2}\right) \widehat{=} 0$. 


\section{ANGULAR MOMENTUM, MASS AND THE FIRST LAW}

In this section we will introduce definitions of mass and angular momentum for type II weakly isolated horizons and derive the first law of black hole mechanics in this context. As in the non-rotating case treated in [4], we will use a Hamiltonian framework.

This section is divided into five parts. In the first, we make appropriate restrictions on the phase space to enable the introduction of a useful notion of angular momentum. The second sub-section considers the issue of defining Hamiltonians generating canonical transformations corresponding to space-time diffeomorphisms and provides criteria for their existence. In the third sub-section, we specialize this discussion to the case where the diffeomorphism reduces to a rotational symmetry at the horizon and show the corresponding Hamiltonian does indeed exist. This Hamiltonian is interpreted as the horizon angular momentum. The fourth sub-section considers space-time diffeomorphisms which can be interpreted as timetranslations and analyzes the issue of whether their induced action on the phase space is Hamiltonian (i.e., preserves the symplectic structure). In contrast to the rotational case, the answer is not always in the affirmative: As in [4, the necessary and sufficient condition for the evolution to be Hamiltonian is precisely that the first law holds. Thus, for every space-time vector field $t^{a}$ which generates a Hamiltonian evolution, there is an associated energy $E_{\Delta}^{(t)}$ and angular momentum $J_{\Delta}$ satisfying the first law. The last sub-section shows that, in the Einstein-Maxwell case, there is a natural way to select a preferred class of evolution vector fields $t_{0}^{a}$ for which $E_{\Delta}^{\left(t_{0}\right)}$ can be interpreted as the horizon mass $M_{\Delta}$. The corresponding first law is then the canonical generalization of the standard first law of black hole mechanics to the context of rotating, weakly isolated horizons.

\section{A. The Phase Space of Rigidly Rotating Horizons}

Physical observables such as energy and angular momentum are naturally associated with symmetries: energy is associated with time-translations and angular momentum with rigid rotations. For example, in the familiar construction of the ADM energy, one first introduces a vector field $t^{a}$ in space-time which asymptotically approaches a time-translation symmetry of the fixed flat metric at infinity and constructs the Hamiltonian $H_{t}$ generating the corresponding time-evolution in the phase space. Since Einstein's theory is generally covariant, "on shell" (i.e. when the constraints are satisfied), the Hamiltonian reduces to 2-surface integrals on the boundaries of the Cauchy surface under consideration. Under the standard assumption that there are no internal boundaries, the on-shell value $E_{\infty}^{(t)}$ of the Hamiltonian is thus given just by a 2-surface integral at infinity, which is interpreted as the total ADM energy of space-time with respect to the asymptotic time-translation of $t^{a}$. Likewise, to define the total angular momentum of space-time, one considers a space-time vector field $\varphi^{a}$ which approaches a rigid rotation of the asymptotic metric at infinity. The angular momentum $J_{\infty}^{(\varphi)}$ is then the surface integral at infinity giving the on-shell value of the Hamiltonian generating rotations along $\varphi^{a}$. To define energy and angular momentum of an isolated horizon, it is therefore natural to examine Hamiltonians which generate appropriate symmetries at the horizon. 
Let us begin with angular momentum. In the above procedure, while there is considerable freedom in the choice of $\varphi^{a}$, these vector fields must approach a fixed rotational Killing field $\phi^{a}$ of the universal flat metric at infinity. This condition plays a key role in the standard proof of the existence of a Hamiltonian generating the corresponding motions in phase space. More importantly, the requirement has a direct physical origin. Angular momentum is not a scalar quantity in physical theories and has several independent components. By fixing one particular axial symmetry across all asymptotically flat space-times, one effectively guarantees the same component of angular momentum is calculated for all space-times. If $\varphi^{a}$ were to approach different rotational Killing fields of the flat background in different space-times, even if one could construct the corresponding conserved quantity, it would be difficult to interpret it physically.

To define angular momentum of weakly isolated horizons, it would be natural to start with vector fields $\varphi^{a}$ which approach a rotational symmetry on the horizon. Recall however that, in contrast to infinity, weakly isolated horizons do not have a universal metric and, furthermore, the metrics in class III isolated horizons need not admit any rotational symmetry at all. Therefore, it is natural first to restrict ourselves to class II, i.e., axi-symmetric, weakly isolated horizons. Note however that, even in this case, the metric on the horizon is not universal, whence a priori we do not have a fixed rotational vector field $\phi^{a}$ on $\Delta$ that the space-time vector fields $\varphi^{a}$ can be required to approach. Therefore, for a meaningful comparison of horizon angular momenta of different space-times, it is convenient to introduce a fixed rotational vector field $\phi^{a}$ on the horizon and admit only those space-times in the phase space which have this $\phi^{a}$ as the horizon symmetry. We will do so.

Thus, let us now fix a vector field $\phi^{a}$ on the inner boundary $\Delta$ of $\mathcal{M}$ such that: (i) it has vanishing Lie bracket with $\left[\ell^{a}\right]$, (ii) vanishes on exactly two generators of $\Delta$, and (iii) has closed, circular orbits of affine length $2 \pi$. For calculational convenience, we will also insist that it be tangent to the past boundary $S_{\Delta}^{-}$of the horizon. Like the equivalence class $\left[\ell^{a}\right]$, this $\phi^{a}$ will now be regarded as an extra structure fixed on $\Delta$ once and for all. The phase space will now consist of the sub-manifold $\mathbb{\Gamma}_{\phi}$ of the covariant phase space $\mathbb{\Gamma}$ (of section 【IO), consisting of those asymptotically flat solutions $\left(e_{I}^{a}, A_{a I}{ }^{J}, \mathbf{A}_{a}\right)$ to the field equations for which $\left(\Delta,\left[\ell^{a}\right], \phi^{a}\right)$ is a type II horizon with $\phi^{a}$ as its rotational symmetry. (Thus, $\mathcal{L}_{\phi} \ell^{a} \in\left[\ell^{a}\right], \mathcal{L}_{\phi} q_{a b} \widehat{=} 0, \mathcal{L}_{\phi} \omega_{a} \widehat{=} 0$ and $\mathcal{L}_{\phi} F_{a b} \widehat{=} 0$.) We will refer to $\mathbb{\Gamma}_{\phi}$ as the phase space of rigidly rotating horizons. In the next two sub-sections we will show that on $\mathbb{\Gamma}_{\phi}$ we can use the standard strategy of defining conserved quantities (outlined in the beginning of this sub-section) and arrive at a definition of the horizon angular-momentum $J_{\Delta}$.

\section{B. Existence of Hamiltonian Generating Space-time Diffeomorphisms}

Fix a vector field $W^{a}$ in each space-time in $\mathbb{\Gamma}_{\phi}$ such that the diffeomorphisms it generates preserve the boundary conditions both at spatial infinity and at the horizon. As discussed below, the Lie derivatives of $\left(e_{I}^{a}, A_{a I}{ }^{J}, \mathbf{A}_{a}\right)$ by $W^{a}$ define a vector field $\delta_{W}$ on $\mathbb{\Gamma}_{\phi}$. The key question of this sub-section is: Is $\delta_{W}$ Hamiltonian? Or, alternatively, does the Lie derivative of the symplectic structure along $\delta_{W}$ vanish? We will find a necessary and sufficient condition for the answers to these questions to be in the affirmative. This result will then be used to define the horizon angular momentum and energy in the next two sub-sections. In this 
sub-section, we will allow $W^{a}$ to vary from one space-time to another. Since we fixed the rotational symmetry field $\phi^{a}$ on $\Delta$, this generality is not necessary for the definition of angular momentum. However, as we will see in Section IVD, it is essential to the definition of energy because, in contrast to spatial infinity, the near horizon geometry can vary from one space-time to another. This complication makes the question of existence for certain Hamiltonians rather more subtle in the presence of a weakly isolated horizon.

For calculational simplicity, it is useful to introduce a universal foliation of the horizon by 2-spheres, although our final results do not depend on it. We saw in section IIB that a non-extremal, weakly isolated horizon admits a natural foliation of this type. But we would like to incorporate the extremal case as well. Let us therefore define the leaves of the foliation as the rigid translations of the past boundary $S_{\Delta}^{-}$of $\Delta$ along any element of the equivalence class $\left[\ell^{a}\right]$. (In the non-extremal case, these are precisely the level surfaces of the function $\psi$ appearing in the symplectic structure and this foliation coincides with the preferred foliation if and only if the past boundary $S_{\Delta}^{-}$is a leaf of the preferred foliation.) The practical advantage of introducing such a foliation is that it allows us to decompose a vector field $W^{a}$ on the horizon in to vertical and horizontal components, exactly as in (3.5).

Consider, then, a smooth assignment of a vector field $W^{a}$ on $\mathcal{M}$ to each space-time in the phase space $\mathbb{\Gamma}_{\phi}$ such that, at infinity, $W^{a}$ is an asymptotic symmetry, and on the horizon $W^{a}$ is tangential to $\Delta$, and

$$
W^{a}=B_{W} \ell^{a}+h_{W}^{a},
$$

with $\mathcal{L}_{\ell} h_{W}^{a}=0$ and $B_{W}$ a constantf on $\Delta$. The motion in phase space associated to the diffeomorphism along $W^{a}$ is given simply by the Lie derivative:

$$
\delta_{W} \Sigma_{a b I}^{J}=\mathcal{L}_{W} \Sigma_{a b I}{ }^{J}, \quad \delta_{W} A_{a I}{ }^{J}=\mathcal{L}_{W} A_{a I}{ }^{J} \quad \text { and } \quad \delta_{W} \mathbf{A}_{a}=\mathcal{L}_{W} \mathbf{A}_{a} .
$$

When the background fields $\left(\Sigma_{a b I}^{J}, A_{a I}^{J}, \mathbf{A}_{a}\right)$ satisfy the field equations of Einstein-Maxwell theory, one can easily verify that $\delta_{W}$ satisfies the linearized equations of motion. It therefore represents a tangent vector field on covariant phase space. This vector field generates a canonical transformation if it preserves the symplectic structure, i.e., if $\mathcal{L}_{\delta_{W}} \Omega=0$. Equivalently, $\delta_{W}$ is a canonical transformation if and only if there exists a Hamiltonian function $H_{W}$ on phase space such that

$$
\delta H_{W}=\Omega\left(\delta, \delta_{W}\right)
$$

for all tangent vectors $\delta$ to phase space. (As with any generally covariant theory, one expects the Hamiltonian, if it exists, will consist only of surface terms. We will see that this expectation is correct.)

Using the symplectic structure (2.16) and dropping terms which vanish when the equations of motion hold and $\delta$ satisfies the linearized equations of motion, the right side of (4.1) becomes

\footnotetext{
${ }^{5}$ As we saw in Section III, if $\kappa_{(\ell)}=0, B_{W}$ need not be constant on $\Delta$ for $W^{a}$ to define a symmetry of that horizon. However, our purpose here is to consider a smooth assignment of symmetry vectors to many different horizons. By continuity in phase space, therefore, $B_{W}$ should be constant in this case as well.
} 


$$
\begin{aligned}
\Omega\left(\delta, \delta_{W}\right)= & \left.\left.\frac{1}{16 \pi G} \int_{M} \mathrm{~d} \operatorname{Tr}[\delta A \wedge(W\lrcorner \Sigma)+(W\lrcorner A\right) \delta \Sigma\right] \\
& -\frac{1}{8 \pi G} \oint_{S_{\Delta}}(\delta \psi)\left(\delta_{W}^{2} \epsilon\right)-\left(\delta_{W} \psi\right)\left(\delta^{2} \epsilon\right) \\
& \left.\left.-\frac{1}{4 \pi} \int_{M} \mathrm{~d}[\delta \mathbf{A} \wedge(W\lrcorner \star \mathbf{F})+(W\lrcorner \mathbf{A}\right) \delta(\star \mathbf{F})\right] \\
& -\frac{1}{4 \pi} \oint_{S_{\Delta}}(\delta \chi)\left(\delta_{W}(\star \mathbf{F})\right)-\left(\delta_{W} \chi\right)(\delta(\star \mathbf{F})) .
\end{aligned}
$$

The remaining bulk terms reduce to surface integrals on $S_{\Delta}$ and $S_{\infty}$. The integrals on $S_{\infty}$ are the usual ones and are not the main focus of this subsection. On the other hand, the surface terms at $S_{\Delta}$ arising from the bulk integrals, together with those already present in (4.2), will provide the critical test of whether $\delta_{W}$ defines a symmetry of the symplectic structure; we will focus on these.

The first issue one must address is the definition of $\delta_{W} \psi$ and $\delta_{W} \chi$. Since $\psi$ and $\chi$ are uniquely determined by the triplet $\left(e_{I}^{a}, A_{a I}^{J}, \mathbf{A}_{a}\right)$ at the horizon (see (2.14) and (2.15)), $\delta_{W} \psi$ and $\delta_{W} \chi$ are completely unambiguous. However, their explicit expressions involve a subtlety. For definiteness, let us consider $\delta_{W} \psi$. One may first be tempted to set $\delta_{W} \psi=\mathcal{L}_{W} \psi$. However, recall the definition (2.14) of $\psi$ required $\psi$ to vanish on $S_{\Delta}^{-}$. The naive definition of $\delta_{W} \psi$ generally does not preserve this condition. Hence the naive expression is incorrect and must be modified to ensure that $\delta_{W} \psi$ vanishes on $S_{\Delta}^{-}$. To address this problem, let us proceed systematically and return to definition (2.14) of $\psi$. Using the second part to the definition (2.14) of $\psi$ and the properties $\mathcal{L}_{W} \ell^{a}=0$ and $\mathcal{L}_{W} \omega_{a} \widehat{=} 0$ of $W$, it follows that

$$
\mathcal{L}_{\ell}\left(\delta_{W} \psi\right) \widehat{=} 0
$$

Since $\delta_{W} \psi$ must vanish on $S_{\Delta}^{-}$, we conclude $\delta_{W} \psi \widehat{=} 0$ on all of $\Delta$. A similar argument applies to $\chi$, whence we conclude

$$
\delta_{W} \psi=0=\delta_{W} \chi
$$

Note that the subtlety arose because $\psi$ and $\chi$ are potentials for physical fields. Since all other terms in (4.2) involve the fields themselves, there is no further subtlety in defining the action of $\delta_{W}$; the action is given simply by the Lie derivative.

The remainder of the calculation is straightforward. The surface terms in (4.2) arising from the bulk integrals can be expressed in terms of $\omega_{a}$ and ${ }^{2} \epsilon_{a b}$. The final result can be conveniently expressed as

$$
\begin{aligned}
\Omega\left(\delta, \delta_{W}\right)= & \left.\left.\frac{-1}{8 \pi G} \oint_{S_{\Delta}} \delta\left[\left(h_{W}\right\lrcorner \omega\right)^{2} \epsilon\right]-\left(\delta h_{W}\right\lrcorner \omega\right)^{2} \epsilon+\kappa_{(W)} \delta^{2} \epsilon \\
& \left.\left.-\frac{1}{4 \pi} \oint_{S_{\Delta}} \delta\left[\left(h_{W}\right\lrcorner \mathbf{A}\right) \star \mathbf{F}\right]-\left(\delta h_{W}\right\lrcorner \mathbf{A}\right) \star \mathbf{F}-\Phi_{(W)} \delta(\star \mathbf{F}) \\
& \left.\left.+\frac{1}{16 \pi G} \oint_{S_{\infty}} \operatorname{Tr}[\delta A \wedge(W\lrcorner \Sigma)+(W\lrcorner A\right) \delta \Sigma\right] \\
& \left.\left.-\frac{1}{4 \pi} \oint_{S_{\infty}} \delta \mathbf{A} \wedge(W\lrcorner \star \mathbf{F}\right)+(W\lrcorner \mathbf{A}\right) \delta(\star \mathbf{F}),
\end{aligned}
$$

where $\Phi_{(W)}$ denotes the electric potential of the horizon relative to the vertical component of $W^{a}$. The right side of this result consists of integrals both at the horizon and at infinity. If the 
Hamiltonian $H_{W}$ is to exist, then the right side of (4.3) must be equal to the exact variation of some expression. As is well-known, the surface integrals at infinity can themselves be written as exact variations whenever $W^{a}$ becomes a symmetry of the asymptotic metric at infinity. However, there is no a priori reason why the surface integral at the horizon is an exact variation. Thus, somewhat surprisingly, although the evolution generated by $W^{a}$ does yield a flow on the phase space $\mathbb{\Gamma}_{\phi}$, this flow need not be Hamiltonian. The necessary and sufficient condition for it to be Hamiltonian is precisely that the surface integral on $S_{\Delta}$ in (4.3) equals $\delta H_{\Delta}^{(W)}$ for some function $H_{\Delta}^{(W)}$ on $\mathbb{\Gamma}_{\phi}$ (depending only on values of horizon fields).

\section{Angular Momentum}

To define angular momentum, let us assign a vector field $\varphi^{a}$ on $\mathcal{M}$ to each space-time in $\mathbb{\Gamma}_{\phi}$ such that its restriction to $\Delta$ is given by our fixed rotational vector field $\phi^{a}$. Since we are primarily interested only in the horizon angular momentum, to avoid the unnecessary analysis of the terms at infinity, let us further require that $\varphi^{a}$ vanishes outside some compact neighborhood of the horizon. (We will relax this requirement after obtaining the expression of the horizon angular momentum.) Then, setting $W^{a}=\varphi^{a}$, (4.3) simplifies: the term at infinity vanishes and $\delta h_{W}^{a}$ can be set to zero. Thus, we now have

$$
\left.\left.\Omega\left(\delta, \delta_{\varphi}\right)=\frac{-1}{8 \pi G} \oint_{S_{\Delta}} \delta[(\phi\lrcorner \omega)^{2} \epsilon\right]-\frac{1}{4 \pi} \oint_{S_{\Delta}} \delta[(\phi\lrcorner \mathbf{A}) \star \mathbf{F}\right] .
$$

The right side of this expression is clearly the variation of an integral over $S_{\Delta}$. Therefore, we conclude the Hamiltonian generating motions along $\varphi^{a}$ does indeed exist. Moreover, that Hamiltonian will consist of a single surface term at $\Delta$ whose value may be taken to define the angular momentum $J_{\Delta}$ of the horizon:

$$
\left.\left.J_{\Delta}:=H_{\Delta}^{\phi}=-\frac{1}{8 \pi G} \oint_{S_{\Delta}}(\phi\lrcorner \omega\right)^{2} \epsilon-\frac{1}{4 \pi} \oint_{S_{\Delta}}(\phi\lrcorner \mathbf{A}\right) \star \mathbf{F},
$$

where the integral can be evaluated on any cross-section of $\Delta$. This definition is manifestly (quasi-)local to the horizon. Since $\omega_{a}$ and ${ }^{2} \epsilon_{a b}$ are invariant under the permissible tetrad rotations, the gravitational term is invariant under internal Lorentz rotations at the horizon. Using the fact that $\mathcal{L}_{\phi}(\star \mathbf{F})_{a b} \widehat{=} 0$ and the Maxwell equations, it is easy to check that the Maxwell term is also gauge invariant. Finally, note that, in contrast to the standard angular momentum expressions at infinity [13], the horizon angular momentum $J_{\Delta}$ includes contributions from both the gravitational and electro-magnetic fields. In this respect the right side of (4.4) is completely analogous to the expression of the horizon energy derived in [1] in the non-rotating case.

It is natural to ask if the horizon angular momentum can be expressed directly in terms of space-time curvature at $\Delta$. The intuition derived from the Newman-Penrose framework suggests that the gravitational contribution to the angular momentum is encoded in the component $\operatorname{Im}\left[\Psi_{2}\right]$ of the Weyl curvature. We will now show that his rule of thumb is explicitly realized in the present construction. Since $\phi^{a}$ is a Killing vector of the intrinsic horizon geometry, it is also a symmetry of the area element ${ }^{2} \epsilon_{a b}$. Thus, we find $\mathcal{L}_{\phi}{ }^{2} \epsilon=$ 
$\left.\mathrm{d}(\phi\lrcorner^{2} \epsilon\right)=0$, from which it follows that $\left.\phi\right\lrcorner^{2} \epsilon=\mathrm{d} f$ for some smooth function $f$ satisfying $\mathcal{L}_{\ell} f=0$ on the horizon. Now, since $\phi^{a}$ is tangent to $S_{\Delta}$, we have

$$
\left.\left.\oint_{S_{\Delta}}(\phi\lrcorner \omega\right)^{2} \epsilon=\oint_{S_{\Delta}} \omega \wedge(\phi\lrcorner{ }^{2} \epsilon\right)=\oint_{S_{\Delta}} 2 f \operatorname{Im}\left[\Psi_{2}\right]^{2} \epsilon
$$

where we have performed an integration by parts in the last step. Next, consider the electromagnetic term in (4.4). Since $\mathcal{L}_{\phi} \star \mathbf{F}=0$ and $\mathrm{d} \star \mathbf{F} \hat{=} 0$, it follows that $\left.\phi\right\lrcorner \star \mathbf{F}=\mathrm{d} g$ for some smooth function $g$ satisfying $\overleftarrow{\mathcal{L}_{\ell} g} \widehat{=} 0$ on the horizon. Hence, using an $\overleftarrow{\text { identical }}$ argument as above we can replace the term $(\phi\lrcorner \mathbf{A})$ involving the electromagnetic potential by the Newman-Penrose component $\phi_{1}$ of the Maxwell field (given by $\phi_{1}=-\frac{1}{4}^{2} \epsilon^{a b}\left[(\star \mathbf{F})_{a b}+\right.$ $\left.\left.i \mathbf{F}_{a b}\right]\right)$. Thus, we can re-express the horizon angular momentum (4.4) as

$$
J_{\Delta}=-\frac{1}{4 \pi G} \oint_{S_{\Delta}} f \operatorname{Im}\left[\Psi_{2}\right]{ }^{2} \epsilon+\frac{1}{2 \pi} \oint_{S_{\Delta}} g \operatorname{Im}\left[\phi_{1}\right]{ }^{2} \epsilon .
$$

Since the integrands in (4.5) are Lie-dragged by $\ell$, the full expression can be projected to the 2-sphere $\hat{S}$ of generators of $\Delta$.

Having the precise definition at hand, we can now ask for its relation with other notions of angular momentum available in the literature.

First suppose that there is no Maxwell (or any other field) in a neighborhood of $\Delta$ and $\varphi^{a}$ is in fact a Killing field of a specific geometry in our phase-space $\mathbb{\Gamma}_{\phi}$ in this neighborhood. Then, one could also define the angular momentum via Komar integral. To analyze the relation between the two definitions, first consider the 1-form $n_{a}$ on $\Delta$ which is normal to the foliation by $S_{\Delta}$ and is normalized with $\ell^{a} n_{a} \widehat{=}-1$ everywhere. It is easy to show $\ell^{a} \nabla_{a} n_{b} \widehat{=} \omega_{b}$, where $\nabla_{a}$ denotes the space-time connection. Using this relation in the gravitational contribution to the angular momentum definition (4.4), one finds

$$
\left.\left.\left.J_{\Delta}=-\frac{1}{8 \pi G} \oint_{S_{\Delta}}(\phi\lrcorner \nabla_{\ell} n\right)^{2} \epsilon=\frac{1}{8 \pi G} \oint_{S_{\Delta}}\left(\nabla_{\ell} \phi\right\lrcorner n\right)^{2} \epsilon=\frac{1}{16 \pi G} \oint_{S_{\Delta}}(\ell\lrcorner \mathrm{d} \varphi\right) \cdot n^{2} \epsilon,
$$

where we have used the Killing property of $\varphi^{a}$ in the last equality. By re-writing the last integral in terms of the the space-time dual of the 2 -form $\mathrm{d} \varphi$, we obtain

$$
J_{\Delta}=\frac{1}{8 \pi G} \oint_{S_{\Delta}} \star \mathrm{d} \varphi
$$

The right side is precisely the Komar angular momentum. Thus, in any space-time in which $\phi^{a}$ can be extended to a space-time Killing field $\varphi^{a}$ in a neighborhood of $\Delta$, the gravitational contribution to $J_{\Delta}$ agrees with the usual Komar expression defined by $\varphi^{a}$. As at infinity, this is an exact agreement, not just a proportionality. Finally note that, even in presence of Maxwell fields on the horizon, the above discussion establishes the equality of the Komar integral with the gravitational term in (4.4).

A second definition of angular momentum is the one associated with infinity [13]. However, the integral at infinity represents the total angular momentum, including that in the radiation fields outside the horizon. Therefore, in general, one does not expect the two to agree. Indeed, a priori, it is not clear to which component of the angular momentum at infinity we should compare $J_{\Delta}$. However, this problem disappears if the space-time under 
consideration admits a global, rotational Killing field $\varphi^{a}$ (with $\mathcal{L}_{\varphi} \mathbf{F}_{a b}=0$ on $\mathcal{M}$ ), whose restriction to $\Delta$ is given by $\phi^{a}$. Let us therefore consider this case.

Now, since $\varphi^{a}$ is everywhere a Killing field of the space-time under consideration, the vector field $\delta_{\varphi}$ on the phase space can only define an infinitesimal gauge transformation. However, such a gauge transformation defines a degenerate direction of the symplectic structure. Thus, we have

$$
\delta H_{\phi}=\Omega\left(\delta, \delta_{\phi}\right)=0
$$

for all tangent vectors $\delta$ at any axially symmetric point $\left(e_{I}^{a}, A_{a I}{ }^{J}, \mathbf{A}_{a}\right)$ of the phase space. Therefore, on any connected component of the phase-space $\mathbb{\Gamma}_{\phi}$ consisting of space-times which admit $\varphi^{a}$ as a Killing field, $H_{\phi}$ must be a constant. Note, however, that with $G=c=1, H_{\phi}$ has dimensions of angular momentum ((length $\left.)^{2}\right)$ while the theory has no dimensionful constant with that dimension (recall: the cosmological constant is zero). Therefore, Hamiltonian itself must vanish. Finally, from (4.3) it follows that, on shell, the Hamiltonian consists only of two surface terms:

$$
H_{\phi}=J_{\Delta}-J_{\infty}
$$

We therefore conclude: if the space-time admits a global rotational Killing field $\varphi^{a}$ which reduces to $\phi^{a}$ on $\Delta$, then

$$
J_{\Delta}=J_{\infty}
$$

where $J_{\Delta}$ is the total horizon angular momentum (defined by $\phi^{a}$ ).

At first this result (4.6) is rather surprising, both mathematically and physically, because one would have expected $J_{\infty}$ and $J_{\Delta}$ to differ from each other by the angular momentum in Maxwell field outside the horizon. However, a closer examination shows that the result is to be expected but for rather subtle reasons. Let us first consider the mathematical aspect. In the axi-symmetric case, it is well-known that $J_{\infty}$ is given by the Komar integral $J_{\infty}^{\mathrm{K}}$ defined by $\varphi^{a}$ at infinity [14]. We saw above that the gravitational contribution to $J_{\Delta}$ equals the Komar integral $J_{\Delta}^{\mathrm{K}}$ evaluated at the horizon. It turns out that, using the Cartan identity, the $\varphi^{a}$-angular momentum in the bulk electromagnetic field -i.e., the integral $\int T_{a b} \varphi^{a} d S^{b}$ over any partial Cauchy surface extending from the horizon to infinity - can in fact be expressed as surface integrals. The term at infinity vanishes because of the fall-off conditions while the term at the horizon is precisely the electromagnetic contribution to $J_{\Delta}$. Thus, the bulk electromagnetic contribution to $\varphi$-component of angular momentum is already contained in $J_{\Delta}$ through the Maxwell horizon term. Physically, one can understand the situation as follows. In general, if $\Delta$ extends in the future all the way to $i^{+}$, the horizon angular momentum $J_{\Delta}$ is to be thought of as the angular momentum "left over at $i^{+}$after allowing for radiation". (This is completely analogous to the situation with the horizon mass analyzed in detail in [3].) Now, if $\varphi$ is a Killing vector, there is no radiation of the $\varphi$-component of angular momentum, whence the $\varphi$-component evaluated at $i^{+}$is the same as that evaluated at $i^{o}$, whence $J_{\Delta}=J_{\infty}$.

We will conclude with three remarks.

1. Let us restrict ourselves to class I (i.e. spherically symmetric) weakly isolated horizons. In this case, $q_{a b}, \omega_{a}$ and $\mathbf{A}_{a}$ are Lie-dragged by all three rotational vector fields. Hence, the 
pull-backs to the spherical sections of $\omega_{a}$ and $\mathbf{A}_{a}$ must vanish and the integrand of (4.4) therefore vanishes. Thus, as one would physically expect, $J_{\Delta}$ vanishes on all class I horizons.

2. The vector field $\delta_{\varphi}$ determines the Hamiltonian $H_{\varphi}$ only up to an additive constant. How was this freedom fixed in (4.4)? In the case where the cosmological constant vanishes, this constant can be fixed to zero because there is no parameter available in the theory with the correct dimension. Even when the cosmological constant is not zero, the freedom can still be eliminated, e.g., simply by the physical requirement that horizon angular momentum should vanish in class I. As we just showed, $J_{\Delta}$ satisfies this condition. Thus, the additive constant is fixed in a natural fashion.

3. An important question for this framework is whether the event horizon of a KerrNewman black hole is a rigidly rotating isolated horizon and, if so, whether the above definition of angular momentum reproduces the standard result. The answer, in both cases, is in the affirmative. Being Killing horizons, these event horizons are in particular weakly isolated horizons and the axi-symmetry of the ambient space-time defines the field $\phi^{a}$ on $\Delta$. Thus, these are rigidly rotating isolated horizons. Finally, the discussion above shows that in this case $J_{\Delta}$ equals $M a$, the standard angular momentum $J_{\infty}$ defined at infinity. Thus (as with the horizon mass in the non-rotating case [四]), our horizon angular momentum expression (4.4) contains not only the "bare" angular momentum one may naively associate with the horizon but also the contributions from the electromagnetic hair outside.

\section{The First Law}

To state the first law of black hole mechanics for isolated horizons, we must first define horizon energy. As with angular momentum in the previous sub-section, we will base our definition of energy on the Hamiltonian generating translations along an appropriate symmetry of the horizon. However, which particular symmetry $W^{a}=t^{a}$ would correspond to the desired time-translations is not immediately clear. Therefore, we will begin with an arbitrary symmetry field $t^{a}$. At the horizon, then, it only has to satisfy

$$
t^{a}+\Omega_{(t)} \phi^{a} \in\left[\ell^{a}\right] \quad \text { or } \quad t^{a}=B_{(\ell, t)} \ell^{a}-\Omega_{(t)} \phi^{a}
$$

for some constants $\Omega_{(t)}, B_{(\ell, t)}$ on $\Delta$. (The latter depends not only on the specific choice of $t^{a}$ but also of $\ell^{a} \in\left[\ell^{a}\right]$.) The constant $\Omega_{(t)}$ will be referred to as the angular velocity of the horizon relative to $t^{a}$. Note that both the specific element $B_{(\ell, t)} \ell^{a}$ of $\left[\ell^{a}\right]$ and $\Omega_{(t)}$, determined by $t^{a}$, can depend on the dynamical fields $\left(e_{I}^{a}, A_{a I}{ }^{J}, \mathbf{A}_{a}\right)$; in the numerical relativity terminology, we are considering "live" evolution vector fields $t^{a}$. This generality is essential in particular because the physically appropriate angular velocity $\Omega_{(t)}$ of $\Delta$ will vary from one space-time to another. To summarize, to each point in the phase space $\mathbb{\Gamma}_{\phi}$, we assign a vector field $t^{a}$ on $\mathcal{M}$ satisfying (4.7) on $\Delta$, allowing the vector field to vary from one point of the phase space to another.

To analyze the question of whether $\delta_{t}$ is a Hamiltonian vector field on $\mathbb{\Gamma}_{\phi}$, we must determine whether the right side of Hamilton's equations (4.1) is the exact variation of some Hamiltonian. We have already calculated the quantity in question for an arbitrary vector field $W^{a}$ in (4.3). Since the surface terms at infinity are not central to this discussion, as in the angular momentum calculation above, for simplicity let us first assume that $t^{a}$ 
vanishes outside of some compact neighborhood of $\Delta$. The only potential obstruction to $\delta_{t}$ being Hamiltonian lies in the horizon surface term of (4.3). Being linear in $\delta_{t}$, this surface term defines a 1 -form $X_{\Delta}^{t}$ on phase space. From the right side of (4.3), $X_{\Delta}^{t}$ can be expressed as

$$
\begin{aligned}
\left.\left.X_{\Delta}^{t}(\delta)=\frac{1}{8 \pi G} \oint_{S_{\Delta}} \delta\left[\left(-\Omega_{(t)} \phi\right\lrcorner \omega\right)^{2} \epsilon\right]-\left[\delta\left(-\Omega_{(t)} \phi\right)\right\lrcorner \omega\right]^{2} \epsilon+\kappa_{(t)} \delta^{2} \epsilon \\
\left.\left.\quad+\frac{1}{4 \pi} \oint_{S_{\Delta}} \delta\left[\left(-\Omega_{(t)} \phi\right\lrcorner \mathbf{A}\right) \star \mathbf{F}\right]-\left[\delta\left(-\Omega_{(t)} \phi\right)\right\lrcorner \mathbf{A}\right] \star \mathbf{F}+\Phi_{(t)} \delta(\star \mathbf{F})
\end{aligned}
$$

where we have decomposed $t$ as in (3.5) with $h_{t}=-\Omega_{(t)} \phi^{a}$. Let us begin with the first integral. Since $\phi$ is fixed once and for all on $\Delta$, the variation $\delta$ in the second term only affects $\Omega_{(t)}$. Therefore, the second term will simply cancel the part of the first term where the variation hits $\Omega_{(t)}$ and we can therefore write the first two terms together as

$$
\left.\frac{1}{8 \pi G} \oint_{S_{\Delta}}-\Omega_{(t)} \delta[(\phi\lrcorner \omega)^{2} \epsilon\right]
$$

However, the quantity $\Omega_{(t)}$ is constant over $\Delta$ and may therefore be pulled outside the integral. The remaining integrand is exactly the $\delta$-variation of the gravitational contribution to the angular momentum (4.4). Analogous procedure can be applied to the second (i.e., electro-magnetic) term of (4.8), likewise yielding the electro-magnetic contribution to $J_{\Delta}$. Furthermore, since the surface gravity and electro-static potential are both constant over $\Delta$, we can pull them outside the respective integrals and (4.8) reduces to

$$
X_{\Delta}^{t}(\delta)=\frac{\kappa_{(t)}}{8 \pi G} \delta a_{\Delta}+\Omega_{(t)} \delta J_{\Delta}+\Phi_{(t)} \delta \mathbf{Q}_{\Delta}
$$

Note that the right side of (4.9) is strongly reminiscent of the first law of black hole mechanics. However, at this stage of our analysis, it only provides an explicit expression of the 1 -form $X_{\Delta}^{t}$ on $\mathbb{\Gamma}_{\phi}$. The condition that $\delta_{t}$ be Hamiltonian is simply that the 1 -form $X_{\Delta}^{t}$ is closed:

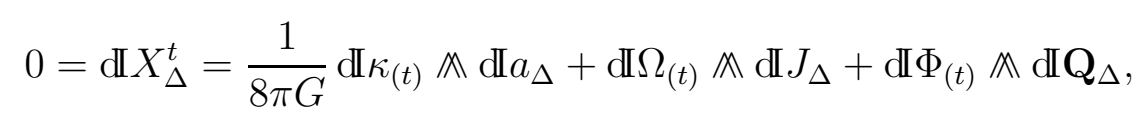

where $\mathbb{d}$ and $\mathbb{A}$ denote the exterior derivative and exterior product on the (infinitedimensional) phase space $\mathbb{\Gamma}_{\phi}$. This simple relation leads to some startling consequences which we now discuss.

A priori, the horizon value of $t^{a}$ can vary from one space-time to another in any smooth fashion; for each such choice, we obtain a flow on the phase space. Eq (4.10) implies that most of these flows fail to preserve the symplectic structure. To begin with, for the flow to be Hamiltonian, the surface gravity $\kappa_{(t)}$, the angular velocity $\Omega_{(t)}$ and the electric potential $\Phi_{(t)}$ can be functions only of the area $a_{\Delta}$, angular momentum $J_{\Delta}$ and the charge $\mathbf{Q}_{\Delta}$. 9 Other factors such as the local geometry (i.e. distortion) of the horizon can not affect

\footnotetext{
${ }^{6}$ So far, our construction is very general. For any given physical metric (satisfying our boundary
} 
these "extrinsic parameters". Moreover, these parameters must satisfy certain non-trivial relations:

$$
\begin{aligned}
& \frac{\partial \kappa_{(t)}\left(a_{\Delta}, J_{\Delta}, \mathbf{Q}_{\Delta}\right)}{\partial J_{\Delta}}=8 \pi G \frac{\partial \Omega_{(t)}\left(a_{\Delta}, J_{\Delta}, \mathbf{Q}_{\Delta}\right)}{\partial a_{\Delta}} \\
& \frac{\partial \kappa_{(t)}\left(a_{\Delta}, J_{\Delta}, \mathbf{Q}_{\Delta}\right)}{\partial \mathbf{Q}_{\Delta}}=8 \pi G \frac{\partial \Phi_{(t)}\left(a_{\Delta}, J_{\Delta}, \mathbf{Q}_{\Delta}\right)}{\partial a_{\Delta}} \\
& \frac{\partial \Omega_{(t)}\left(a_{\Delta}, J_{\Delta}, \mathbf{Q}_{\Delta}\right)}{\partial \mathbf{Q}_{\Delta}}=\frac{\partial \Phi_{(t)}\left(a_{\Delta}, J_{\Delta}, \mathbf{Q}_{\Delta}\right)}{\partial J_{\Delta}}
\end{aligned}
$$

Now, on any given point $\left(e_{I}^{a}, A_{a I}^{J}, \mathbf{A}_{a}\right)$ in the phase space, $\kappa_{(t)}, \Omega_{(t)}$ and $\Phi_{(t)}$ are completely determined by the horizon value of $t^{a}$. Conversely, at any given phase space point, $\kappa_{(t)}$ uniquely determines the vertical component of $t^{a}$ and $\Omega_{(t)}$, the horizontal component. Therefore, relations (4.11) constrain the permissible choices of the assignment of $t^{a}$ to each space-time in $\mathbb{\Gamma}_{\phi}$. These strong restrictions are the necessary and sufficient conditions for the flow generated by $\delta_{t}$ on $\mathbb{\Gamma}_{\phi}$ to be Hamiltonian.

Thus, the restriction to the horizon of a permissible evolution field $t^{a}$ is determined by this remarkably small set of parameters of the horizon. Note, however, that these arguments do not provide a specific choice of functions $\kappa_{(t)}$ and $\Omega_{(t)}$ of the horizon parameters $a_{\Delta}, J_{\Delta}, \mathbf{Q}_{\Delta}$. Correspondingly, for any choice of these functions satisfying (4.11), the Hamiltonian generating evolution along the corresponding $t^{a}$ is guaranteed to exist. (The term at infinity is already an exact variation and is therefore not relevant to our discussion.) The horizon surface term in that Hamiltonian is a natural measure of the energy $E_{\Delta}^{t}$ of the horizon relative to the evolution field $t^{a}$. By virtue of (4.8) and the calculations above, the energy $E_{\Delta}^{t}$ is a function only of $a_{\Delta}, J_{\Delta} \mathbf{Q}_{\Delta}$ and satisfies

$$
\delta E_{\Delta}^{t}=\frac{\kappa_{(t)}}{8 \pi G} \delta a_{\Delta}+\Omega_{(t)} \delta J_{\Delta}+\Phi_{(t)} \delta \mathbf{Q}_{\Delta}
$$

This is our generalized first law, which holds for all weakly isolated horizons. The analogy to the usual first law of black hole mechanics is clear.

conditions) we can choose an $[\ell]$ so that $(\Delta,[\ell])$ is an isolated horizon with $\kappa_{(\ell)}=0$ and another equivalence class $\left[\ell^{\prime}\right]$ with $\kappa_{\left(\ell^{\prime}\right)} \neq 0$. Hence, for any values of the triplet $\left(a_{\Delta}, J_{\Delta}, \mathbf{Q}_{\Delta}\right)$, and an evolution field $t^{a} \widehat{=} B \ell^{a}-\Omega \phi^{a}$ (with $B \neq 0$ ), there is a phase space point with $\kappa_{(t)} \equiv B \kappa_{(\ell)}=0$ and another with $\kappa_{(t)} \neq 0$. Therefore, irrespective of the choice of $t^{a}$, the evolution will fail to be Hamiltonian unless we remove the spurious redundancy in the phase space. This can be naturally accomplished as follows. Denote by $\kappa_{\mathrm{KN}}\left(a_{\Delta}, J_{\Delta}, \mathbf{Q}_{\Delta}\right)$ the function of the three intrinsic horizon parameters which yields surface gravity in the Kerr-Newman family. We will excise those points from our phase space for which one of $\left(\kappa_{(\ell)}, \kappa_{\mathrm{KN}}\right)$ vanishes but the other does not. This excision could have been carried already in Section $\amalg \mathrm{IO}$ when we introduced the phase space. We did not do so because the reason behind the excision becomes clear only after (4.10). Note that the phase space continues to contain all Kerr-Newman solutions, including the extremal ones, after the excision. 
Let us summarize. A priori there is freedom to assign an evolution vector field $t^{a}$ on $\mathcal{M}$ to every point in the phase space $\mathbb{\Gamma}_{\phi}$ in any smooth fashion; each assignment provides an evolution flow $\delta_{t}$ on $\mathbb{\Gamma}_{\phi}$. However, most of these flows fail to preserve the symplectic structure. They do so if and only if the assignment is such that (4.10) (or, equivalently, (4.11)) holds. While this is a severe restriction on the assignment of $t^{a}$, as discussed below, it still leaves considerable freedom in the choice of the assignment. For each such "permissible" $t^{a}$, there is a well-defined horizon energy and the first law holds. Thus, there is a precise sense in which the first law (4.12) is a necessary and sufficient condition for the $\delta_{t}$-evolution to be Hamiltonian.

Since there is generally no canonical choice of a single, "correct" evolution field at the horizon, there is no canonical notion of the horizon energy. While $E_{\Delta}^{t}$ has a direct Hamiltonian interpretation in the phase space $\mathbb{\Gamma}_{\phi}$, for a general permissible $t^{a}$ it does not admit an obvious space-time interpretation. In the next sub-section, we will show that a canonical choice of $t^{a}$ can be made using no-hair theorems in Einstein-Maxwell theory. The corresponding $E_{\Delta}^{t}$ can be interpreted as the horizon mass.

We will conclude with two remarks.

1. There is a constructive procedure to obtain permissible vector fields. Choose any smooth function $\kappa_{0}$ of $a_{\Delta}, J_{\Delta}, \mathbf{Q}_{\Delta}$ satisfying the following regularity condition for each choice of $J_{\Delta}, \mathbf{Q}_{\Delta}$ : the integrals

$$
\int_{a_{0}}^{\infty} \mathrm{d} a_{\Delta} \frac{\partial \kappa_{0}}{\partial J_{\Delta}} \quad \text { and } \quad \int_{a_{0}}^{\infty} \mathrm{d} a_{\Delta} \frac{\partial \kappa_{0}}{\partial \mathbf{Q}_{\Delta}}
$$

converge to well-defined functions of $J_{\Delta}$ and $\mathbf{Q}_{\Delta}$, with $a_{0}=4 \pi \sqrt{Q^{4}+4 J^{2}}$ (and $\kappa_{0}$ vanishes if and only if $\kappa_{\mathrm{KN}}$ vanishes). Then, integrating the first of Eqs (4.11) with respect to $a_{\Delta}$ and requiring $\Omega\left(a_{\Delta}, J_{\Delta}, \mathbf{Q}_{\Delta}\right)$ to tend to zero as $a_{\Delta}$ tends to infinity for any fixed values of $J_{\Delta}, \mathbf{Q}_{\Delta}$, we obtain a unique function $\Omega_{0}\left(a_{\Delta}, J_{\Delta}, \mathbf{Q}_{\Delta}\right)$. Now, given any point $\left(e_{I}^{a}, A_{a I}{ }^{J}, \mathbf{A}_{a}\right)$ in $\mathbb{\Gamma}_{\phi}$, there is a unique vector field $t^{a}$ on $\Delta$ such that $\kappa_{(t)}=\kappa_{0}$ and $\Omega_{(t)}=\Omega_{0}$ (namely, $t^{a} \bumpeq B_{0} \ell^{a}+\Omega_{0} \phi^{a}$ where $B_{0}$ is given by $\left.\kappa_{0}=B_{0} \kappa_{(\ell)}\right)$. Finally, we can integrate the second of Eqs (4.11) with respect to $a_{\Delta}$ and require that $\Phi_{(t)}$ tends to zero as $a_{\Delta}$ tends to infinity (keeping $J_{\Delta}$ and $\mathbf{Q}_{\Delta}$ fixed), we obtain a function $\Phi_{(t)}$ of $a_{\Delta}, J_{\Delta}, \mathbf{Q}_{\Delta}$ which satisfies the second and the third of Eqs (4.11). Thus, given a sufficiently regular function $\kappa_{0}$ of $a_{\Delta}, J_{\Delta}, \mathbf{Q}_{\Delta}$, we can integrate (4.11) and, using physically motivated conditions to determine integration constants, obtain an admissible evolution field $t^{a}$ and electric potential $\Phi_{(t)}$ on $\Delta$.

2. If we now drop the restriction that $t^{a}$ vanish outside a finite neighborhood of $\Delta$ in $\mathcal{M}$ but require instead that it asymptotically approach a time-translation Killing field of the fixed flat metric at infinity, we find that the total Hamiltonian has two terms:

$$
H_{t}=E_{\infty}^{t}-E_{\Delta}^{t}
$$

where $E_{\infty}^{t}$ is the ADM energy. Again, general arguments from symplectic geometry imply that $H_{t}$ is constant on each connected component of stationary solutions, assuming of course that $t^{a}$ coincides with the stationary Killing field in these space-times. As in the case of angular momentum, we can argue that the value of this constant can only be zero in the Einstein-Maxwell theory. Thus, in each stationary space-time, the horizon energy $E_{\Delta}^{t}$ equals the ADM energy at infinity. 


\section{E. Horizon Mass}

The procedure that led us to the definition of the horizon energy $E_{\Delta}^{t}$ is the same as the one used at spatial infinity to define the ADM energy. However, the boundary conditions at infinity are such that the asymptotic value of the evolution field $t^{a}$ must coincide with one of the time translation Killing fields of the fixed flat metric, whence the space of viable timetranslations at infinity is three-dimensional (corresponding to the unit space-like hyperboloid in the tangent space of $i^{o}$ ). On the other hand, at the inner isolated horizon boundary, the physically appropriate $t^{a}$ necessarily varies from one space-time to another. In particular, if the horizon is non-rotating, $t^{a}$ points along the null normal to the horizon while if it is rotating, it has a component also along the rotational symmetry $\phi^{a}$.

In the usual treatments of the first law, one restricts oneself to perturbations of stationary backgrounds. Consequently, one can single out a preferred time translation from the 3parameter family, adapted to the rest frame of the black hole. The corresponding ADM energy is then also the ADM mass. It is natural to ask if we can similarly single out a canonical time translation also at the horizon and introduce the notion of horizon mass. Note that this task is significantly more difficult than the corresponding task at spatial infinity first because the permissible time translations at the horizon form an infinite dimensional family rather than three and second because, on physical grounds mentioned above, this canonical time translation at the horizon must vary from one space-time to another. Nonetheless, because of the remarkably strong restrictions on the extrinsic parameters $\kappa_{(t)}$, $\Omega_{(t)}$ and $\Phi_{(t)}$ and thanks to the no-hair theorems, there is a natural solution to this problem.

We have just seen in the previous sub-section that the restriction of any evolution vector field $t^{a}$ to the horizon is determined by $\kappa_{(t)}$ and $\Omega_{(t)}$ and if the $t^{a}$-evolution is to define a Hamiltonian flow on $\mathbb{\Gamma}_{\phi}, \kappa_{(t)}$ and $\Omega_{(t)}$ can only be functions of $a_{\Delta}, J_{\Delta}$ and $\mathbf{Q}_{\Delta}$. These three quantities may be regarded as the independent parameters of the horizon. The problem of defining a canonical time-translation on the horizon therefore reduces to that of making a canonical choice of the two functions $\kappa_{(t)}\left(a_{\Delta}, J_{\Delta}, \mathbf{Q}_{\Delta}\right)$ and $\Omega_{(t)}\left(a_{\Delta}, J_{\Delta}, \mathbf{Q}_{\Delta}\right)$. Now, event horizon of a Kerr-Newman black hole is in particular a weakly isolated horizon. It is natural, on these stationary space-times, to choose the evolution field to coincide with the stationary Killing field. This choice selects for us specific functions of the three parameters:

$$
\kappa=\frac{R_{\Delta}^{4}-G^{2}\left(\mathbf{Q}_{\Delta}^{4}+4 J_{\Delta}^{2}\right)}{2 R_{\Delta}^{3} \sqrt{\left(R_{\Delta}^{2}+G \mathbf{Q}_{\Delta}^{2}\right)^{2}+4 G^{2} J_{\Delta}^{2}}} \quad \text { and } \quad \Omega=\frac{2 G J_{\Delta}}{R_{\Delta} \sqrt{\left(R_{\Delta}^{2}+G \mathbf{Q}_{\Delta}^{2}\right)^{2}+4 G^{2} J_{\Delta}^{2}}}
$$

where, as before, $a_{\Delta}=4 \pi R_{\Delta}^{2}$. Now, although the choice (4.13) was made only for stationary cases, since there is exactly one Kerr-Newman solution for each set of isolated horizon parameters, this choice uniquely fixes the two functions. Thus, we can select a canonical time-translation on any isolated horizon by requiring that the surface gravity and the angular velocity it defines be given by (4.13). (This procedure is unambiguous because $R_{\Delta}, J_{\Delta}, \mathrm{Q}_{\Delta}$ are determined by $\left(e_{I}^{a}, A_{a I}^{J}, \mathbf{A}_{a}\right)$ without any reference to $t^{a}$.) We will make this choice and denote by $t_{0}^{a}$ any time-translation whose restriction to the horizon coincides with this canonical choice. The conditions (4.11) then require us to partially gauge fix the Maxwell field such that 


$$
\Phi=\frac{\mathbf{Q}_{\Delta}}{R_{\Delta}} \frac{R_{\Delta}^{2}+G \mathbf{Q}_{\Delta}^{2}}{\sqrt{\left(R_{\Delta}^{2}+G \mathbf{Q}_{\Delta}^{2}\right)^{2}+4 G^{2} J_{\Delta}^{2}}} .
$$

(Again this is the same value which the electro-static potential of the horizon takes in a Kerr-Newman solution.) The evolution generated by such a live vector field $t_{0}^{a}$ is then guaranteed to be Hamiltonian. We can then "integrate" the expression (4.12) of the first law to find the horizon energy $E_{\Delta}^{t}$. Setting it equal to the mass $M_{\Delta}$, we find:

$$
M_{\Delta}=\frac{\sqrt{\left(R_{\Delta}^{2}+G \mathbf{Q}_{\Delta}^{2}\right)^{2}+4 G^{2} J_{\Delta}^{2}}}{2 G R_{\Delta}} .
$$

Again, this gives the usual ADM mass for the Kerr-Newman solutions. However, this definition applies to all isolated horizons including those which admit radiation in the exterior region. Therefore, in general, $M_{\Delta}$ differs from the ADM mass $M_{\mathrm{ADM}}$ due to the energy in the radiation field. In the Einstein-Maxwell theory under consideration, if the weakly isolated horizon were to extend all the way to $i^{+}$in the distant future, $M_{\Delta}$ can be thought of as the future limit of the Bondi mass at $i^{+}$[3]. If the space-time is globally stationary, there is no flux of radiation across future null infinity $\mathcal{I}^{+}$whence the future limit of the Bondi mass coincides with the ADM mass. Finally, we wish to emphasize that we did not simply postulate (4.15); it was systematically derived using Hamiltonian methods.

With this (quasi-)local definition of the horizon mass, for any preferred time-translation $t_{0}^{a}$, we have a canonical generalized first law

$$
\delta M_{\Delta}=\frac{\kappa}{8 \pi G} \delta a_{\Delta}+\Omega \delta J_{\Delta}+\Phi \delta \mathbf{Q}_{\Delta}
$$

applicable to all weakly isolated horizons in Einstein-Maxwell theory.

\section{DISCUSSION}

In this paper, we gave a definition of angular momentum $J_{\Delta}$ for all rigidly rotating isolated horizons. The definition is (quasi-)local to the horizon; it makes no reference to infinity at all. In contrast to the usual angular momentum expression [13 at infinity, $J_{\Delta}$ has an explicit contribution from the Maxwell field on the horizon. If the space-time in a neighborhood of the horizon is axi-symmetric and the matter fields vanish in that region, $J_{\Delta}$ equals the usual Komar integral formula, evaluated at the horizon. If the space-time is globally axi-symmetric, irrespective of whether there is Maxwell field on the horizon, $J_{\Delta}$ equals the total angular momentum at infinity. If the weakly isolated horizon extends all the way to $i^{+}$, we can regard $J_{\Delta}$ as the future limit of angular momentum along future null infinity $\mathcal{I}^{+}$. While these properties of the $J_{\Delta}$ are very similar to those of horizon mass $M_{\Delta}$, there is also an interesting difference: Whereas in presence of radiation in the exterior region $M_{\Delta}$ is always different from $M_{\mathrm{ADM}}$, if the radiation field is axi-symmetric, $J_{\Delta}$ equals the angular momentum at infinity (along the rotational Killing vector).

We have also generalized the standard first law of black hole mechanics. Although

the final form (4.16) of this law is identical to that of the standard one, there are some 
important differences. First, our law is applicable to all space-times which admit an isolated horizon inner boundary, including those which allow radiation arbitrarily close to the horizon. Second, all quantities and variations that enter the first law are defined locally at the horizon. In standard treatments, the physical meaning and appropriateness of variations is not as clear because some quantities such as area, surface gravity and the angular velocity of the horizon are defined at the horizon while others, like energy, angular momentum (and sometimes even the electric scalar potential) are evaluated at infinity. Third, other treatments based on a Hamiltonian framework [1] often critically use the bifurcate 2-surface which does not exist in the extremal case. Therefore, extremal black holes are often excluded from the first law. The present analysis never makes reference to bifurcate surfaces (which do not exist in physical space-times resulting from gravitational collapse). Therefore, our discussion of the first law holds also in the extremal case. Finally, with obvious modifications of boundary conditions at infinity, our analysis includes cosmological horizons where thermodynamic considerations are also applicable [15].

Perhaps the most important aspect of this analysis is that it sheds new light on the 'origin' of the first law: as in the non-rotating case treated in [4], it arose as a necessary and sufficient condition for the existence of a Hamiltonian generating time evolution. A new feature of our framework is the existence of an infinite family of first laws corresponding to the infinite family of evolution "permissible" vector fields $t^{a}$ (i.e., vector fields satisfying the necessary and sufficient condition (4.10) for the evolution to be Hamiltonian.) In the Einstein-Maxwell case, using the no-hair results, one can select a canonical "live" evolution field $t_{o}^{a}$. Correspondingly, there is a canonical notion of energy which can be interpreted as the horizon mass $M_{\Delta}$ and hence a canonical first law. In more general theories which allow hairy black holes, a canonical horizon mass can not be defined on the full phase space. Yet, even in this case, the isolated horizon framework is directly useful: it enables one to relate properties of these hairy black holes to those of the corresponding solitons [8].

Finally, our Lagrangian and Hamiltonian frameworks are based on real tetrads and Lorentz (rather than self-dual) connections. It is therefore quite straightforward to extend our analysis to any space-time dimension.

\section{ACKNOWLEDGMENTS}

We would like to thank Alejandro Corichi, Olaf Dreyer, Steve Fairhurst, Sean Hayward, Badri Krishnan, Daniel Sudarsky, Bob Wald and Jacek Wisniewski for discussions. This work was supported in part by the NSF grants PHY00-90091, INT97-225514, PHY97-34871, the Polish Committee for Scientific Research grant 2 P03B 060, the Eberly research funds of Penn State and the Albert Einstein Institute of the Max Planck Society. CB was supported in part by a Braddock Fellowship.

\section{APPENDIX A: GENERAL HAMILTONIANS}

In section [IV, we constructed Hamiltonians generating the infinitesimal symmetries of rigidly rotating weakly isolated horizons. While this is the most interesting case from a 
physical perspective, for completeness, one may also wish to consider more general spacetime vector fields $W^{a}$ which are tangential to $\Delta$ and preserve the fixed equivalence class $\left[\ell^{a}\right]$ thereon and ask: Can any of these lead to Hamiltonian evolutions on the full phase space $\mathbb{\Gamma}$ ? In this appendix we will analyze this issue. While a general $W^{a}$ will of course not lead to a Hamiltonian evolution, there is an interesting sub-class which does. It may be useful in future investigations.

Let us begin with an assignment of a vector field $W^{a}$ to each space-time in the full phase space $\mathbb{\Gamma}$ which preserves $\left(\Delta,\left[\ell^{a}\right]\right)$ but is not necessarily an infinitesimal symmetry in the sense of Section $\mathbb{R V}$ (i.e., does not necessarily preserve $\left(q_{a b}, \omega_{a}, \mathbf{A}_{a}\right)$ ). As in Section $\mathbb{\nabla}$, we can decompose $W^{a}$ into vertical and horizontal parts:

$$
W^{a}=B_{W} \ell^{a}+h_{W}^{a}
$$

with $\mathcal{L}_{\ell} h_{W} \hat{=} 0$. Note that the condition $\mathcal{L}_{W} \ell^{a} \in\left[\ell^{a}\right]$ implies $B_{W}$ is constrained only by $\mathcal{L}_{\ell} B_{W} \bumpeq 0$. The variations $\delta_{W} \psi$ and $\delta_{W} \chi$ of the potentials become

$$
\delta_{W} \psi=\left(B_{W}-B_{W}^{-}\right) \kappa_{(\ell)}, \quad \text { and } \quad \delta_{W} \chi=\left(B_{W}-B_{W}^{-}\right) \Phi_{(\ell)}
$$

where $B_{W}^{-}$denotes the restriction of $B_{W}$ to the past boundary $S_{\Delta}^{-}$of the horizon.

We can now calculate the action of the symplectic structure on the vector field $\delta_{W}$ on $\mathbb{\Gamma}$. The derivation of (1.2) from (2.16) did not depend on any way on $W^{a}$ defining a symmetry of the horizon. We can therefore begin at that point and substitute the new definitions of $\delta_{W} \psi$ and $\delta_{W} \chi$ to find the analog of (4.3) for generic horizon diffeomorphisms. If fact, the result will be quite similar: For any tangent vector field $\delta$ on $\mathbb{\Gamma}$, we will have

$$
\begin{aligned}
\Omega\left(\delta, \delta_{W}\right)= & \left.\left.\frac{-1}{8 \pi G} \oint_{S_{\Delta}} \delta\left[\left(h_{W}\right\lrcorner \omega\right)^{2} \epsilon\right]-\left(\delta h_{W}\right\lrcorner \omega\right)^{2} \epsilon+\kappa_{\left(W^{-}\right)} \delta^{2} \epsilon \\
& \left.\left.-\frac{1}{4 \pi} \oint_{S_{\Delta}} \delta\left[\left(h_{W}\right\lrcorner \mathbf{A}\right) \star \mathbf{F}\right]-\left(\delta h_{W}\right\lrcorner \mathbf{A}\right) \star \mathbf{F}-\Phi_{\left(W^{-}\right)} \delta(\star \mathbf{F}) \\
& \left.\left.+\frac{1}{16 \pi G} \oint_{S_{\infty}} \operatorname{Tr}[\delta A \wedge(W\lrcorner \Sigma)+(W\lrcorner A\right) \delta \Sigma\right] \\
& \left.\left.-\frac{1}{4 \pi} \oint_{S_{\infty}} \delta \mathbf{A} \wedge(W\lrcorner \star \mathbf{F}\right)+(W\lrcorner \mathbf{A}\right) \delta(\star \mathbf{F}) .
\end{aligned}
$$

The only difference here is that $\kappa_{\left(W^{-}\right)}$denotes the surface gravity relative to the vertical part $B_{W} \ell^{a}$ of $W^{a}$, evaluated at $S_{\Delta}^{-}$, and similarly for the electric potential $\Phi_{\left(W^{-}\right)}$. (On other sections $S_{\Delta}$ of the horizon, the surface gravity and electric potential may take other values.) Since the right side contains several terms which are not exact variations, there are a number of possible barriers to the existence of a Hamiltonian generating motions along $W^{a}$. However, as we will now show, there are a couple of interesting cases where the required Hamiltonian does exists.

Consider first the case when $W$ is purely horizontal at the horizon, $W^{a} \hat{=} h_{W}^{a}$, with, moreover, $h_{W}^{a}$ fixed (i.e., independent of the space-time under consideration). Thus, $W^{a}$ generates a fixed diffeomorphism on $\Delta$ which preserves the family of cross-sections $S_{\Delta}$ which, however, need not be a rotation. Since we are interested in the horizon structure, let us also assume that $W^{a}$ vanishes in a neighborhood of infinity. Then, the integrals at infinity in 
(A2) vanish and only the first two terms in each of the integrals at $S_{\Delta}$ survive. Moreover, the second term in each integral vanishes because $\delta h_{W}^{a}$ is zero. Thus, the flow generated by these $W^{a}$ preserves the symplectic structure and the corresponding Hamiltonian is given by

$$
\left.\left.H_{h_{W}}:=\frac{-1}{8 \pi G} \oint_{S_{\Delta}}\left(h_{W}\right\lrcorner \omega\right)^{2} \epsilon-\frac{1}{4 \pi} \oint_{S_{\Delta}}\left(h_{W}\right\lrcorner \mathbf{A}\right) \star \mathbf{F} .
$$

Note that this integral has the same form as the integral (4.4) used to define the angular momentum in section IV. However, this formula applies to a general weakly isolated horizon, which need not be rigidly rotating. Moreover, the horizontal vector field $h_{W}^{a}$ is arbitrary; it need not be a Killing vector of $q_{a b}$ and indeed it need not even preserve the area-form ${ }^{2} \epsilon$. (Consequently, we can not re-express (A3) in terms of curvatures as in (4.5).) One might hope this formula could be used to define angular momentum in some sense for generic weakly isolated horizons. However, while the phase space interpretation of (A3) is clear, if $h_{W}^{a}$ does not generate rotations, its space-time interpretation is quite obscure.

Finally there is another family of vector fields $W^{a}$ for which the flow $\delta_{W}$ on $\mathbb{\Gamma}$ is Hamiltonian, although physically less interesting. Let $W^{a}$ be purely vertical at the horizon, $W^{a} \hat{=} B_{W} \ell^{a}$, with $B_{W}^{-} \hat{=} 0$. As before, since we want to focus on the horizon terms, let us assume that $W^{a}$ vanishes outside some neighborhood of $\Delta$. Then, terms at infinity in (A2) vanish and because $h_{W}^{a} \hat{=} 0$, the first two terms in each of the surface integrals at $S_{\Delta}$ also vanish. Finally, since $B_{W}^{-} \hat{=} 0$, these remaining terms also vanish. Thus, the entire right side of $\mathrm{A2}$ vanishes. Consequently, the associated $\delta_{W}$ is a degenerate direction of the symplectic structure, whence, from the perspective of the Hamiltonian framework, $\delta_{W}$ generates a gauge transformation. Such transformations have no direct physical interest and may be quotiented out of the algebra of kinematically allowable diffeomorphisms. Indeed, since any $W^{a}$ of the form described above can be written uniquely as a combination of one of these gauge transformations and another kinematically allowable vector field with $B_{W}$ constant over $\Delta$, we may restrict attention to the later case without any loss of generality. 


\section{REFERENCES}

[1] J. W. Bardeen, B. Carter and S. W. Hawking, The four laws of black hole mechanics, Commun. Math. Phys. 31161 (1973);

B. Carter, Black hole equilibrium states, Black Holes, ed B DeWitt and C DeWitt (Gordon and Breach: New York) (1973);

R. M. Wald, Quantum Field Theory in Curved Spacetime and Black Hole Thermodynamics (University of Chicago Press) (1994);

M. Heusler, Black Hole Uniqueness Theorems (cambridge University Press, Cambridge) (1996).

[2] A. Ashtekar, A. Corichi and K. Krasnov, Isolated horizons: the classical phase space. Adv. Theor. Math. Phys. 3 418-471 (2000); gr-qc/9905089.

[3] A. Ashtekar, C. Beetle and S. Fairhurst, Isolated horizons: a generalization of black hole mechanics, Class. Quantum Grav. 16 L1-L7 (1999); gr-qc/9812065.

A. Ashtekar, C. Beetle and S. Fairhurst, Mechanics of isolated horizons, Class. Quantum Grav. 17 253-298 (2000); gr-qc/9907068.

[4] A. Ashtekar, S. Fairhurst and B. Krishnan, Isolated Horizons: Hamiltonian Evolution and the First Law, Phys. Rev. D62 104025 (2000); gr-qc/0005083.

[5] A. Ashtekar, C. Beetle, O. Dreyer, S. Fairhurst, B. Krishnan, J. Lewandowski and J. Wiśniewski, Generic Isolated Horizons and their Applications, Phys. Rev. Lett., 85, 3564-3567 (2000); gr-qc/0006006;

O. Dreyer, B. Krishnan, E. Schattner and D. Shoemaker (in preparation).

[6] A. Ashtekar, C. Beetle and J. Lewandowski, Geometry of Generic Isolated Horizons, CGPG pre-print (2001).

[7] A. Ashtekar, C. Beetle and J. Lewandowski, Space-time Geometry Near Isolated Horizons, CGPG pre-print (2001).

[8] A. Ashtekar, A. Corichi and D. Sudarsky, Hairy black holes, horizon mass and solitons, Class. Quantum Grav. 18, 919-940 (2001); gr-qc/0011081.

[9] A. Corichi and D. Sudarsky, Mass of colored black holes Phys. Rev. D61 101501 (2000); gr-qc/9912032.

A. Corichi, U. Nucamendi and D. Sudarsky, Einstein-Yang-Mills Isolated Horizons: Phase Space, Mechanics, Hair and Conjectures, Phys. Rev. D62, 044046 (2000); gr-qc/0002078.

[10] A. Ashtekar, J. Baez, A. Corichi, K. Krasnov. Quantum geometry and black hole entropy. Phys. Rev. Lett. 80 904-907 (1998).

[11] A. Ashtekar, J. Baez, K. Krasnov. Quantum geometry of isolated horizons horizons and black hole entropy, Adv. Theo. Math. Phys 4, 1-95 (2000)

[12] B. Krishnan and J. Willis, Private communication (2000).

[13] A. Ashtekar and R. O. Hansen, Unified treatment of spatial and null infinity in general relativity: universal structure, asymptotic symmetries and conserved quantities at spatial infinity, J. Math. Phys. 19, 1542-1566 (1978).

[14] A. Ashtekar and A. Magnon, Conserved quantities in general relativity, J. Math. Phys. 20, 793-800 (1979).

[15] G. Gibbons and S.W. Hawking, Cosmological event horizons, thermodynamics, and particle creation Phys. Rev. D15 2738 (1977). 